\title{
High Z neoclassical transport: application and limitation of analytical formulae for modelling JET experimental parameters
}

\author{
S. Breton ${ }^{1}$, F.J. Casson ${ }^{2}$, C. Bourdelle ${ }^{1}$, C. Angioni ${ }^{3}$, E. Belli ${ }^{4}$, Y. Camenen ${ }^{5}$, \\ J. Citrin ${ }^{6}$, X. Garbet ${ }^{1}$, Y. Sarazin ${ }^{1}$, M. Sertoli ${ }^{2}$, the JET contributors* \\ EUROfusion Consortium, JET, Culham Science Centre, Abingdon, OX14 3DB, UK \\ ${ }^{1}$ CEA, IRFM, F-13108 Saint-Paul-lez-Durance, France. \\ ${ }^{2}$ CCFE, Culham Science Centre, Abingdon, Oxon, OX14 3DB, UK \\ ${ }^{3}$ Max-Planck-Institut für Plasmaphysik, Garching, Germany \\ ${ }^{4}$ General Atomics, PO Box 85608, San Diego, CA 92186-5608, USA \\ ${ }^{5}$ Aix-Marseille Université, CNRS, PIIM UMR 7345, 13397 Marseille Cedex 20, France and \\ ${ }^{6}$ FOM Institute DIFFER: Dutch Institute for Fundamental Energy Research, \\ PO Box 6336, 5600 HH Eindhoven, The Netherlands
}

(*See the author list of "Overview of the JET results in support to ITER" by X.

Litaudon et al. to be published in Nuclear Fusion Special issue: overview and summary reports from the 26th Fusion Energy Conference (Kyoto, Japan, 17-22 October 2016))

\footnotetext{
Heavy impurities, such as tungsten (W), can exhibit strongly poloidally asymmetric density profiles in rotating or radio frequency heated plasmas. In the metallic environment of JET, the poloidal asymmetry of tungsten enhances its neoclassical transport up to an order of magnitude, so that neoclassical convection dominates over turbulent transport in the core. Accounting for asymmetries in neoclassical transport is hence necessary in the integrated modeling framework. The neoclassical drift kinetic code, NEO [E. Belli and J. Candy, Plasma Phys. Control. Fusion P50, 095010 (2008)], includes the impact of poloidal asymmetries on $\mathrm{W}$ transport. However, the computational cost required to run NEO slows down significantly integrated modeling. A previous analytical formulation to describe heavy impurity neoclassical transport in the presence of poloidal asymmetries in specific collisional regimes [C. Angioni and P. Helander, Plasma Phys. Control. Fusion 56, 124001 (2014)] is compared in this work to numerical results from NEO. Within the domain of validity of the formula, the factor for reducing the temperature screening due to poloidal asymmetries had to be empirically adjusted. After adjustment, the modified formula can reproduce NEO results outside of its definition domain, with some limitations: When main ions are in the banana regime, the formula reproduces NEO results whatever the collisionality regime of impurities, provided the poloidal asymmetry is not too large. However, for very strong poloidal asymmetries, agreement requires impurities in the Pfirsch-Schlüter regime. Within the JETTO integrated transport code, the analytical formula combined with the poloidally symmetric neoclassical code NCLASS [W. A. Houlberg. K. C. Shaing. S. P. Hirshman. and M. C. Zarnstorff. Phys. Plasmas 4. 3230 (1997)] predicts the same tungsten prfile as NEO in certain cases, while saving a factor of one thousand in computer time, which can be useful in scoping studies. The parametric depedencies of the temperature screening reduction due to poloidal asymmetries would need to be better characterised for this faster model to be extended to a more general applicability.
} 


\section{INTRODUCTION}

Tungsten (W) was chosen as a Plasma Facing Component because of its high melting point, its low erosion rate and low hydrogen retention. But due to its large charge number $74, \mathrm{~W}$ ions are not fully stripped even in the hot tokamak core, leading to a relatively high degree of line radiation. This means that accumulation of $\mathrm{W}$ in the plasma core can be highly deleterious. Above a certain threshold, this leads to the loss of confinement and eventually disruptions. To avoid central $\mathrm{W}$ accumulation, an accurate understanding of $\mathrm{W}$ transport is a key issue. W transport is both turbulent and neoclassical. In the central region of JET core, W transport has been shown to be mostly neoclassical [1-5], whereas in the outer part the turbulent transport dominates. Due to its large mass $\mathrm{A}=184$, W is subject to a strong centrifugal force when the plasma rotates. This causes poloidal asymmetry in W density. In presence of NBI momentum input, those asymmetries are shown to increase neoclassical W transport by an order of magnitude in JET [5, 6]. Ion Cyclotron Resonance Heating (IRCH) with anisotropic temperature distribution and Radio Frequency heating also impact the poloidal distribution of $\mathrm{W}$ up to a factor of $2[7,8]$. The neoclassical drift kinetic code $\mathrm{NEO}[9,10]$ includes comprehensive treatments of poloidal asymmetries. However the computational cost of running NEO is of the same order of turbulent quasilinear codes such as QuaLiKiz [11] and TGLF [12] when embedded in integrated modeling platforms such as JETTO [13].

The goal of this work is to study an alternative solution applying an analytical formula that describes the impact of poloidal asymmetries on heavy impurity transport. Based on previous works such as[14-16] and especially [17], Angioni and Helander proposed such a formula in [18]. This formula, combined with the neoclassical code NCLASS [19] in which poloidal asymmetries are not included, offers a faster option in integrated modeling. The Angioni Helander formula [18] derives the impurity neoclassical flux with a simplified collision model, valid for main ion in banana regime at low Mach number, impurity in trace limit and in collisional Pfirsch-Schlüter regime. These constraints are not simultaneously fulfilled for all radii in experimental plasmas. It is therefore essential to explore the validity domain of the analytical formulation up to realistic ranges. JET-ILW like parameters from H-mode plasmas are used as an illustration of such realistic conditions. This formula was tested out of its validity domain against NEO for several parameter scans, in order to test its robustness and its limits. At first it was noted that within the validity domain of the formula a factor had to be empirically adjusted. Then the modified formula was compared to NEO outside of its validity range, and it is found to remain valid outside its definition domain. Indeed, considering main ions in the banana regime, it well reproduces NEO results whatever the collisionality regime of impurities, provided the poloidal asymmetry is not too large. Finally, the formula combined with NCLASS is used inside the transport solver JETTO to simulate a discharge, and then the W density profiles predicted are compared the ones obtained with an integrated simulation with NEO, while gaining about a factor 1100 of CPU time.

The analytical formula and its limits are introduced in Section II and are generalized. In section III the formula is tested against NEO. Finally in section IV the formula is tested inside JETTO for two different JET-ILW like H-mode pulses and compared with NEO.

\section{NEOCLASSICAL FORMULA}

This section focuses on the theoretical expression for the neoclassical impurity flux in presence of poloidal asymmetries. The theoretical formula is introduced and the limits in which it is derived are discussed.

\section{A. Neoclassical flux of heavy impurities with poloidally asymmetric density distribution}

Neoclassical impurity theory has been generalized to consider the case of poloidally asymmetric heavy impurity such as $\mathrm{W}[14,16-18,20-22]$. The theory is valid whether the $\mathrm{W}$ localization is caused by centrifugal forces or RF induced temperature anisotropy. The impurity labeled Z has to stay in the trace limit $\left(\alpha=\frac{Z_{Z}^{2} n_{Z}}{Z_{i}^{2} n_{i}} \ll 1\right)$. The impurity also has to be in the Pfirsch-Schlüter regime $\left(\nu_{Z} *>1\right)$ while the main ion, labeled i, is in the banana regime $\left(\nu_{i} *<1\right)$. The normalized collisionality $\nu *$ is defined as $\nu^{*}=\frac{\nu q R}{v_{t h} \epsilon^{3 / 2}}$, with q safety factor, $\mathrm{R}$ flux-surface-center major radius, $\varepsilon=r / R$ with $\mathrm{r}$ minor radius and $\mathrm{R}$ flux-surface-center major radius, $v_{t h}$ thermal velocity and $\nu$ collision frequency of the considered species (for the main ion the dominant collisionality is $\nu_{i i}$ ). With a simplified collision operator valid at large aspect ratio $(\varepsilon \ll 1)$, the neoclassical impurity transport can be written as follows (equation (2) of [22], recalled from [18]). 


$$
\frac{R\left\langle\Gamma_{Z}^{\text {neo }}\right\rangle}{\left\langle n_{Z}\right\rangle}=q^{2} D_{c} Z\left[\left(\frac{1}{Z} \frac{R}{L_{n Z}}-\frac{R}{L_{n i}}+\frac{1}{2} \frac{R}{L_{T i}}\right) P_{A}^{\text {model }}-0.33 P_{B}^{\text {model }} f_{c} \frac{R}{L_{T i}}\right]
$$

with

$$
\begin{aligned}
& P_{A}^{\text {model }}=\frac{1}{2 \varepsilon^{2}} \frac{\left\langle B^{2}\right\rangle}{\left\langle n_{Z}\right\rangle}\left[\left\langle\frac{n_{Z}}{B^{2}}\right\rangle-\left\langle\frac{B^{2}}{n_{Z}}\right\rangle^{-1}\right] \\
& P_{B}^{\text {model }}=\frac{1}{2 \varepsilon^{2}} \frac{\left\langle B^{2}\right\rangle}{\left\langle n_{Z}\right\rangle}\left[\frac{\left\langle n_{Z}\right\rangle}{\left\langle B^{2}\right\rangle}-\left\langle\frac{B^{2}}{n_{Z}}\right\rangle^{-1}\right]
\end{aligned}
$$

$\Gamma_{Z}^{n e o}$ is the neoclassical flux of the impurity charge number Z, B is the magnetic field. The gradient length is defined $\operatorname{as} \frac{1}{L_{X}}=-\frac{\nabla_{r}\langle X\rangle}{\langle X\rangle}$. The label i stands for the main ion, $\mathrm{Z}$ for the impurity. With $f_{c}$ the fraction of circulating particles, <.> means that the quantity is flux surface averaged, and $D_{c}=\frac{\rho_{i}^{2}}{\tau_{i i}}$ classical diffusion coefficient. $\rho^{2}{ }_{i}=\frac{v_{t h i}^{2}}{\left\langle\Omega_{i}^{2}\right\rangle}$ is the squared Larmor radius, $\tau_{i i}=\nu_{i i}^{-1}=3(2 \pi)^{3 / 2} \frac{\epsilon_{0}{ }^{2} m_{i}^{1 / 2} T_{i}^{3 / 2}}{n_{i} e^{4} \ln \Lambda}$ is the main ion-main ion collision time, and the thermal velocity is defined as $v_{t h, i}=\sqrt{2 T_{i} / m_{i}}$ with $T_{i}$ the temperature, $n_{i}$ the density and $m_{i}$ the mass of the ion. e is the electron charge, $\varepsilon_{0}$ is the vacuum permittivity, $\ln \Lambda$ is the Coulomb logarithm defined as $\ln \Lambda=\ln \lambda_{D} / \lambda_{L}$ with $\lambda_{L}=e^{2} / 4 \pi \epsilon_{0} k T_{i}$ and $\lambda_{D}=\sqrt{\epsilon_{0} k T_{i} / n_{i} e^{2}}$ with k the Boltzmann constant.

The structure of equation (1) is very similar to the impurity transport flux without asymmetries derived in shown in [23] and reproduced below in equation (4). When comparing equations (1) and (4) below, one can recognize the diffusive term proportional to $\nabla n_{Z}$, the neoclassical pinch driven by $\nabla n_{i}$, both multiplied by the $P_{A}^{\text {model }}$ factor. Concerning the temperature screening driven by $\nabla T_{i}$, two terms are contributing : the term without asymmetries multiplied by $P_{A}^{\text {model }}$ to which a new term is added, proportional to $P_{B}^{\text {model }}$. This new $P_{B}^{\text {model }}$ term decreases the impact of temperature screening. The $P_{A}^{\text {model }}$ and $P_{B}^{\text {model }}$ factors are both purely geometrical and one can notice that without poloidal asymmetries of the impurity density $n_{Z}$, i.e. $\left\langle n_{Z}\right\rangle=n_{Z}, P_{A}^{\text {model }} \rightarrow 1$ and $P_{B}^{\text {model }}=0$ and one recovers Pfirsch-Schlüter $\mathrm{W}$ flux from equation (4) of [23]. In the case of strong asymmetries, $P_{A}^{\text {model }}$ and $P_{B}^{\text {model }}$ increase ; it means that the impurity transport will be more sensitive to the main ion density gradient, and the benefits from temperature screening will be reduced.

\section{B. Theoretical limits and implications}

\section{Symmetric neoclassical temperature screening and pinch coefficients}

Equation (1) above from[22] (recalled from [18]) is derived with the main ion in the banana regime, and heavy impurities in the Pfirsch-Schlüter regime. Heavy impurities must be present as a trace, i.e. $\alpha=\frac{Z_{Z}^{2} n_{Z}}{Z_{i}^{2} n_{i}} \ll 1$. The main ion are also assumed to remain subsonic, $M_{i}=\sqrt{m_{i} / 2 T_{i}} \omega R<1$, where $\omega$ is an angular frequency of toroidal rotation. The impurity Mach number has no constraint, however one can notice that in present experiments with NBI [5] tungsten Mach number can be high, $M_{W}=M_{i} \sqrt{m_{W} / m_{i}} \geq 1$ (see figure 2c).

In experimental plasmas, the main ions in banana and impurities in the trace limit Pfirsch-Schlüter conditions are not always fulfilled (see figure 3 for example based on a JET-ILW pulse), whereas even in NBI JET cases the bulk ion Mach number remains subsonic. This impacts the numerical value $1 / 2$ in front of $\frac{R}{L_{T i}}$ inside equation (1), which is valid only in certain collisionality regimes according to [23]. Therefore this value has to be tested before starting the comparison between the formula and NEO.

In Wenzel-Sigmar neoclassical formulation [23], neoclassical impurity flux without poloidal asymmetries, $\Gamma_{Z}^{P S}$ with impurity in Pfirsch-Schlüter, is described as follows :

$$
\begin{gathered}
\frac{R \Gamma_{Z}^{P S}}{n_{Z}}=q^{2} D_{c} Z\left[K\left(\frac{1}{Z} \frac{R}{L_{n Z}}-\frac{R}{L_{n i}}\right)-H \frac{R}{L_{T i}}\right] \\
K=1-\frac{0.52 \alpha}{0.59+\alpha+1.34 g^{-2}}
\end{gathered}
$$




$$
\begin{gathered}
H=-0.5+\frac{0.29+0.68 \alpha}{0.59+\alpha+1.34 g^{-2}} \\
\text { With } \alpha=\frac{n_{Z} Z_{Z}^{2}}{n_{i} Z_{i}^{2}} \text { and } g=\nu_{D}^{*} \epsilon^{3 / 2}, \nu_{D}^{*}=\frac{\nu_{D D} q R}{v_{t h, D} \epsilon^{3 / 2}}
\end{gathered}
$$

Two coefficients apply, $\mathrm{H}$ and $\mathrm{K}$, respectively on the ion density gradients (main ion i and impurity $\mathrm{Z}$ ) and the main ion temperature gradient. These coefficients depend on the impurity concentration and charge, as well as on the main ion collisionality. Equation (1), from [22] assumes that $\mathrm{K}=1$ and $\mathrm{H}=-0.5$, which is true for trace impurity in Pfirsch-Schlüter regime and main ion in banana regime. Indeed, having the main ion in banana regime implies that $\nu_{D}^{*}<1$ so g term from equations (5) and (6) tends to 0 , therefore $g^{-2}>>1$. If the impurity concentration is low enough to remain in the trace limit (ie $\alpha<<1$ ) then the combination of the two assumptions (impurity trace and main ion banana regime) lead $K$ to tend to 1 and $H$ to tend to $-1 / 2$. But as seen later on figure 3 for JET-like profiles, Deuterium is typically in the banana regime while $\mathrm{W}$ is in plateau regime across most of the plasma, except at the edge where it is in Pfirsch-Schlüter regime.

To check if it is adequate to keep $\mathrm{K}=1$ and $\mathrm{H}=-0.5$ in the formula, a scan of the main ion (assumed to be Deuterium) collisionality was made with NEO. The Deuterium density is varied over a large range, in order to vary D collisionality. Note that in order to reach Deuterium in deep Pfirch-Schlüter regime, unrealistic values of Deuterium densities are used. The ratio $n_{Z} / n_{D}=10^{-5}$ was kept fixed to maintain the impurity in the trace limit $\frac{n_{Z} Z^{2}}{n_{D}}=1.2 .10^{-2}$ with $Z=34$ the charge of $\mathrm{W}$. Therefore, the $\mathrm{W}$ collisionality is varied from plateau to Pfirsch-Schlüter regimes. JET-like parameters are used based on pulse $n^{\circ} 85308$ time averaged over $10.35 \mathrm{~s}-10.85 \mathrm{~s}$ at mid-radius see appendix A for more details. Figure 1 shows the ratio $\mathrm{H} / \mathrm{K}$ versus Deuterium collisionality $\nu_{D}^{*}$, as it is not possible with $\mathrm{NEO}$ to extract $\mathrm{H}$ and $\mathrm{K}$ from the diffusion coefficient $D_{c}$. Indeed the theoretical formulation of $D_{c}$ is only valid for deep Pfirsch-Schlüter regime, which makes it not reliable for a collisionality scan. On figure 1, NEO H/K is compared with Wenzel-Sigmar formula (equations 5 and 6 above) and with NCLASS ([19]). NCLASS is a neoclassical code based on the fluid moment approach with a simplified collision operator, and does not take into account poloidal asymmetries. NCLASS uses the Hirschman-Sigmar collision operator , based on pitch angle scattering. NEO ([9, 21, 24]) solves the full drift kinetic equation. It provides a first-principle calculation of the transport coefficients directly from the kinetic solution of the distribution function. It uses the full linearized Fokker Planck collision operator, which is more accurate, especially for multi-species collisions. NEO is also reliable in general geometry. Two NEO scans were made, one with adiabatic electrons, and the other with kinetic electrons.

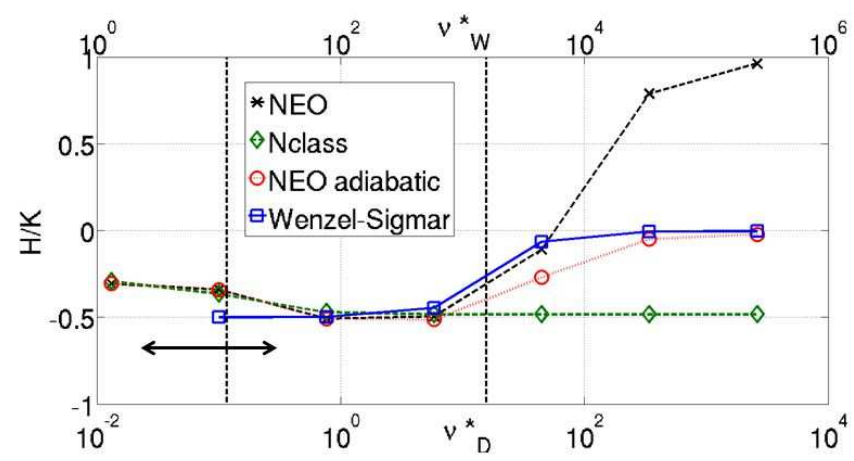

Figure 1: H/K versus D and W collisionalities. Wenzel-Sigmar formulation equations (5) and (6) (solid line squares) NCLASS (diamonds) NEO kinetic electrons (dashed line and crosses) and NEO with adiabatic electrons (dot line and circles). The arrow shows JET-like core values range, see figure 3 below. The parameters used for this scan are : $\mathrm{r} / \mathrm{a}=0.5, \varepsilon=0.16, \mathrm{Z}=34, T_{i}=T_{e}=2.1 \mathrm{keV}, \mathrm{q}=1.18, \mathrm{R}=2.98, \alpha=1.2 .10^{-2}, n_{D}=10^{19} \rightarrow 3.10^{24}$. See Appendix A for the complete NEO input parameters.

Figure 1 can be divided in three zones, separated by vertical dashed lines which correspond to change of collisional regime. The left one indicates that $W$ enters Pfirsch-Schlüter regime, and the right one that Deuterium enters Pfirsch-Schlüter regime. Below $\nu_{D}^{*}=0.1$ in zone $n^{\circ} 1$, Deuterium is in the banana regime and $\mathrm{W}$ is in plateau. When $0.1<\nu_{D}^{*}<15$ in zone $\mathrm{n}^{\circ} 2, \mathrm{~W}$ is in Pfirsch-Schlüter regime and $\mathrm{D}$ is in banana/plateau regime. Above $\nu_{D}^{*}>15$ in zone $\mathrm{n}^{\circ} 3$, both species are in Pfirsch-Schlüter regime. Core range values for the JET-ILW case studied and described 
in section 3 covers zones one and two, with $\mathrm{W}$ in plateau/Pfirsch-Schlüter, and D in banana/plateau (see figure 3). One can start with the first zone : D banana and $\mathrm{W}$ plateau. When the $\mathrm{W}$ is in plateau Wenzel-Sigmar is not applicable. In this zone, both NEO and NCLASS agree very well with each other. In the second zone, the $\mathrm{H} / \mathrm{K}$ value derived in Wenzel-Sigmar in [23] equals $-1 / 2$, NEO and NCLASS first converge towards $-1 / 2$. But as $\nu_{D}^{*}$ enters the third zone, NCLASS remains at -0.5 while $\mathrm{H} / \mathrm{K}$ from [23] and both $\mathrm{NEO}$ curves move away from -0.5 . It means that when D and W go deep in plateau, NCLASS is no longer an adequate model. In the third zone both species are in Pfirsch-Schlüter : in this configuration, according to [25] H/K derived in [23] tends to zero. NEO curve with adiabatic electrons goes to zero, but the NCLASS curve remains at -0.5, and NEO with kinetic electrons goes up to 1. NCLASS behavior comes from the fact that it uses a collision operator which does not include energy scattering, which makes it non-relevant with D in Pfirsch-Schlüter. NEO with kinetic electrons differs from the adiabatic electron case due to the electron coupling. Indeed, at low impurity density, the electron collision frequency with the main ions (i.e. $\nu_{e D}$ ) can become non-neglibible compared with impurity collisional coupling with the main ions (i.e. $\nu_{D W}$ ). This explains the difference betwen kinetic and adiabatic electron NEO runs. NEO with kinetic electrons is the most physical result. For the range of D densities $n_{D}<10^{21} m^{-3}$ (ie $n_{D}<n^{\text {Greenwald }}$ ) NEO and NCLASS predictions of $\mathrm{H} / \mathrm{K}$ transport coefficients practically coincide. However, in the first zone, with Tungsten in the plateau regime, the ratio $\mathrm{H} / \mathrm{K}$ deviates from the $\mathrm{H} / \mathrm{K}=-0.5$. Therefore, one cannot use $\mathrm{H} / \mathrm{K}=-1 / 2$ in the experimentally relevant range (zone 1 and 2), but NCLASS shows to be reliable enough. However the small gradient local models used here (NEO, NCLASS) might not be applicable in the pedestal region.

Two conclusions can be made out of this study of $\mathrm{H} / \mathrm{K}$ term over Deuterium collisionality. First, typical tokamak collisionality ranges values cover a zone where $|\mathrm{H} / \mathrm{K}|$ can be lower than 0.5 . It means that keeping the numerical value in equation (1) is not adequate to describe experimental plasmas. Secondly, NEO and NCLASS give the same results within the experimental parameter range. Therefore the combination of NCLASS with $P_{A}$ and $P_{B}$ formula (equations (1) (2) (3) and [19]) will be compared to NEO with poloidal asymmetries.

Therefore, we assume a generalisation of equation (1) as follows :

$$
\frac{R\left\langle\Gamma_{Z}^{n e o}\right\rangle}{\left\langle n_{Z}\right\rangle}=q^{2} D_{c} Z\left[\left(K \frac{1}{Z} \frac{R}{L_{n Z}}-K \frac{R}{L_{n i}}-H \frac{R}{L_{T i}}\right) P_{A}^{\text {model }}-H_{0} f_{C} P_{B}^{\text {model }} \frac{R}{L_{T i}}\right]
$$

In the limit of main ion in banana regime and $\mathrm{W}$ in the trace limit and Pfirsch-Schlüter regime, $\mathrm{K}=1, \mathrm{H}=-0.5$ and $H_{0}=0.33$ and equation (1) is recovered. The numerical value 0.33 associated with $P_{B}$ term in equation (1) is also valid only for $\mathrm{W}$ in Pfirsch-Schlüter. But as shown later in figure $3 \mathrm{~W}$ is mainly in plateau for the studied JET pulse. Therefore the 0.33 value is generalized to an $H_{0}$ numerical term that will be empirically adjusted based on NEO results.

\section{Extracting $P_{A}$ and $P_{B}$ terms from $N E O$ outputs}

In order to explore the validity of equation (7), one needs to extract the transport coefficients from NEO outputs, and then deduce $P_{A}$ and $P_{B}$ terms calculated with NEO and compare them to their theoretical expressions (equations (2) and (3)). For clarity, equation (7) is re-written : the idea is to gather the terms respectively in front of densities and temperature gradients. The general version of the flux equation, written in equation (8) below is the starting point. The impurity flux is expressed as the sum of a diffusion term and a convective part. The diffusion coefficient is $D_{a s y m}^{\text {model }}$ and the convection velocity coefficient is identified as $V_{a s y m}^{\text {model }}$. The index asym stands for asymmetric, i.e accounting for the effects of poloidal asymmetries. "model" means that the coefficients are based on analytical derivation.

$$
\left\langle\Gamma_{\text {asym, } Z}^{\text {model }}\right\rangle=\frac{D_{a s y m}^{\text {model }} n_{Z}}{R}\left(\frac{R}{L_{n Z}}+\frac{V_{a s y m}^{\text {model }} R}{D_{\text {asym }}^{\text {model }}}\right)
$$

with the pinch velocity term defined as

$$
V_{\text {asym }}^{\text {model }} R=V_{N, \text { asym }}^{\text {model }} \frac{R}{L_{n i}}+V_{T, \text { asym }}^{\text {model }} \frac{R}{L_{T i}}
$$

We define $V_{N, \text { asym }}^{\text {model }}$ and $V_{T, a s y m}^{\text {model }}$ as the convection velocities respectively proportional to $\frac{R}{L_{n i}}$ and $\frac{R}{L_{T i}}$ :

$$
V_{N, \text { asym }}^{\text {model }}=D_{\text {asym }}^{\text {model }} C_{N, \text { asym }}^{\text {model }} \frac{1}{L_{n i}}
$$




$$
V_{T, \text { asym }}^{\text {model }}=D_{\text {asym }}^{\text {model }} C_{T, \text { asym }}^{\text {model }} \frac{1}{L_{T i}}
$$

By identification with equation (7) the coefficients $D_{a s y m}^{\text {model }}, C_{N, \text { asym }}^{\text {model }}$ and $C_{T, \text { asym }}^{\text {model }}$ can be written as follows :

$$
\begin{gathered}
D_{\text {asym }}^{\text {model }}=q^{2} D_{c} K P_{A}^{\text {model }}=D_{\text {sym }}^{\text {model }} P_{A}^{\text {model }} \\
C_{N, \text { asym }}^{\text {model }}=C_{N, \text { sym }}^{\text {model }}=-Z \\
C_{T, \text { asym }}^{\text {model }}=-Z\left(\frac{H}{K}+\frac{H_{0} f_{C} P_{B}^{\text {model }}}{K P_{A}^{\text {model }}}\right)=C_{T, \text { sym }}^{\text {model }}-\frac{Z H_{0} f_{C} P_{B}^{\text {model }}}{K P_{A}^{\text {model }}}
\end{gathered}
$$

In equation (14) $f_{c}=\frac{3\left\langle B^{2}\right\rangle}{4} \int_{0}^{\lambda_{c}} \frac{\lambda d \lambda}{(\sqrt{1-\lambda B})}$ is the fraction of circulating particles with $\lambda$ the pitch angle variable. In this section, we use $f_{c}$ provided by NEO. To recover the transport coefficients in absence of poloidal asymmetries : $D_{\text {sym }}^{\text {model }}, C_{N, \text { sym }}^{\text {model }}$ and $C_{T, \text { sym }}^{\text {model }}$ one just needs to set $P_{A}=1$ and $P_{B}=0$ in equations (12)-(14).

Next we compute the coefficients (10)-(12) from NEO results. The NEO inputs and outputs that are relevant in our simulations are listed in table 1 , the complete inputs at all radii are given in Appendix A. NEO inputs are defined at the outboard midplane, noted as (.) $)_{0}$ in table 1.

$$
\begin{array}{|c|c|}
\hline \text { Inputs } & \text { Outputs } \\
\hline \hline\left(\frac{R}{L_{n i}}\right)_{0},\left(\frac{R}{L_{T i}}\right)_{0},\left(\frac{R}{L_{n Z}}\right)_{0}, n_{W, 0} / n_{i, 0} & \Gamma_{a s y m, Z}^{N E O}, \Gamma_{s y m, Z}^{N E O}, f_{c} \\
\hline
\end{array}
$$

Table I: NEO relevant inputs and outputs used for the simulations

NEO equilibrium coefficients come from the EFIT numerical equilibrium of JET-ILW pulse 85308 at $10.35 \mathrm{~s}$. To extract the diffusion coefficient computed by NEO $D_{a s y m}^{N E O}$, two NEO runs are needed : they are called runs 1 and 2. They share the same inputs, except for the impurity density gradient $\left(\frac{R}{L_{n} z}\right)_{0}$. Sanity checks have been done to ensure that W flux evolves linearly with densities and temperature gradients, therefore only two values of $\left(\frac{R}{L_{n} Z}\right)_{0}$ are needed. To make NEO definition consistent with the gradient of the flux surface average density $\frac{R}{L_{n Z}}$ used in equation (1), the transformation $\left(\frac{R}{L_{n Z}}\right)_{0}=\frac{R}{L_{n Z}} \frac{\left\langle n_{Z}\right\rangle}{n_{Z, 0}}$ is used. Indeed equation (1) uses the average gradient length as defined in [22], therefore a correction factor $\frac{\left\langle n_{Z}\right\rangle}{n_{Z, 0}}$ was added to the NEO gradient length defined at the outboard midplane, as explained in [5]. $\left\langle n_{Z}\right\rangle$ is the $\mathrm{W}$ flux surface averaged density, and $n_{Z, 0}$ is the $\mathrm{W}$ density at the outboard midplane. $\Gamma_{1}$ is the output flux associated with the input $\frac{R}{L_{n Z}}=2$, noted as $\left(\frac{R}{L_{n Z}}\right)_{1} \cdot \Gamma_{2}$ is associated with $\frac{R}{L_{n Z}}=5$, noted as $\left(\frac{R}{L_{n Z}}\right)_{2}$. $D_{\text {asym }}^{N E O}$ is then calculated as shown in equation (15):

$$
D_{\text {asym }}^{N E O}=\frac{R}{\left\langle n_{Z}\right\rangle} \frac{\Gamma_{2}-\Gamma_{1}}{\left(\frac{R}{L_{n Z}}\right)_{2}-\left(\frac{R}{L_{n Z}}\right)_{1}}
$$

We assume that the description of equation (10) is correct, so that $V_{N, \text { asym }}^{N E O}=-Z D_{\text {asym }}^{N E O} \frac{1}{L_{n i}}$.

To extract $V_{T, \text { asym }}^{N E O}$, an extra NEO run is needed, with a different main ion temperature gradient compared with run 1. $\Gamma_{1}$ is the output flux associated with the input $\left(\frac{R}{L_{T_{i}}}\right)_{1}=1, \Gamma_{3}$ is associated with $\left(\frac{R}{L_{T_{i}}}\right)_{3}=0$. It gives equation (16):

$$
V_{T, \text { asym }}^{N E O}=\frac{R}{\left\langle n_{Z}\right\rangle} \frac{\Gamma_{1}-\Gamma_{3}}{\left(\frac{R}{L_{T i}}\right)_{1}-\left(\frac{R}{L_{T i}}\right)_{3}} \frac{1}{L_{T i}}
$$

In total, to compute transport coefficients with poloidal asymmetries $D_{a s y m}^{N E O}$ and $V_{T, \text { asym }}^{N E O}$, and coefficients without poloidal asymmetries, $D_{s y m}^{N E O}$ and $V_{T, s y m}^{N E O}, 6$ NEO runs are needed. 
Since the $P_{A}^{\text {model }}$ geometry coefficient is equivalent to :

$$
P_{A}^{\text {model }}=\frac{D_{\text {asym }}^{\text {model }}}{D_{\text {sym }}^{\text {model }}}
$$

And assuming that equation (7) is an accurate description of NEO results, we define $P_{A}^{N E O}$ as follows :

$$
P_{A}^{N E O}=\frac{D_{\text {asym }}^{N E O}}{D_{\text {sym }}^{N E O}}
$$

Now we have extracted $P_{A}^{N E O}$ from NEO coefficients, we need to do the same with $P_{B}^{N E O}$. However, isolating $H_{0} P_{B}$ alone from NEO transport coefficients is not possible using NEO coefficients (equations (15)-(16)). Therefore the term $Q_{B}^{N E O}$ is defined in equation (20), for it is the simplest coefficient including $H_{0} P_{B}$ that can be extracted. Assuming that equation (7) is a good description of NEO results, $Q_{B}^{\text {model }}$ is defined, extracted using analytical coefficients as shown in equation (19).

$$
\begin{gathered}
Q_{B}^{\text {model }}=-\frac{H_{0} f_{C} P_{B}^{\text {model }}}{H}=\frac{V_{T, \text { sym }}^{\text {model }} P_{A}^{\text {model }}-V_{T, \text { asym }}^{\text {model }}}{V_{T, \text { sym }}^{\text {model }}} \\
Q_{B}^{N E O}=\frac{V_{T, \text { sym }}^{N E O} \frac{D_{a s y m}^{N E O}}{D_{\text {sym }}^{N E O}}-V_{T, \text { asym }}^{N E O}}{V_{T, s y m}^{N E O}}
\end{gathered}
$$

Now we compare the model built from the generalization equation (7) of the analytical formula with NEO.

\section{POLOIDAL ASYMMETRIES IMPACT ON NEOCLASSICAL TRANSPORT : NEO VS FORMULA}

In this section $P_{A}^{N E O}$ and $Q_{B}^{N E O}$ are compared with $P_{A}^{m o d e l}$ and $Q_{B}^{\text {model }}$ for a given case of JET-ILW plasma profiles. Transport coefficients are then reconstructed with a combination of NCLASS runs and geometrical $P_{A}^{m o d e l}$ and $P_{B}^{m o d e l}$ and compared with NEO extracted transport coefficients.

\section{A. Selected JET pulse description and collisionality profiles}

This section focuses on the first test of the robustness of the model. The comparison between NEO and analytical $P_{A}^{\text {model }}$ and $Q_{B}^{\text {model }}$ is based on JET-ILW parameters from the baseline H-mode shot $85308\left(I_{P}=2.5 M A\right.$ and $\left.\mathrm{B}=2.7 \mathrm{~T}\right)$, also presented in [6]. The heating power of this discharge is $19.1 \mathrm{MW}$ of NBI. Be $\left(n_{B e} / n_{D}=2.9 .10^{-2}\right)$, and Hydrogen $\left(n_{H} / n_{D}=9.5 .10^{-2}\right)$ are present in the NEO simulations. The $\mathrm{W}$ concentration is arbitrarily chosen so that $n_{W} / n_{D}=10^{-5}$ and $\mathrm{W}$ remains a trace specie. Other dimensionless quantities are shown in table 2 . Figure 2 shows the main JET based input profiles, and figure 3 shows the collisionalities calculated with these inputs. Table 2 gives the main quantities that are used for NEO runs at three radial locations, with r/a defined as the ratio of the mid-plane averaged minor radius $r$, to the mid-plane minor radius $r$ at the last closed flux surface a. Other NEO inputs are listed in Appendix A. In this section, unless specified otherwise, NEO resolution is the follows : 21 theta poloidal gridpoints, 19 extensions in pitch-angle Legendre polynomial, and 10 Laguerre polynomials. These resolutions were checked to be sufficient at the extrema of our scans.

\begin{tabular}{|c|c|c|c|c|c|c|c|c|}
\hline $\mathrm{r} / \mathrm{a}$ & $R / L_{T_{D}}=R / L_{T_{e}}$ & $R / L_{n_{e}}$ & $Z_{W}$ & $Z_{\text {eff }}$ & $\mathrm{q}$ & $n_{W} / n_{D}$ & $M_{D}$ & $M_{W}$ \\
\hline \hline 0.1 & 2.4 & 1 & 44 & 1.39 & 0.9 & $10^{-5}$ & 0.19 & 1.82 \\
\hline 0.4 & 5.2 & 1.4 & 38 & 1.37 & 1 & $10^{-5}$ & 0.18 & 1.78 \\
\hline 0.8 & 10.4 & 2.8 & 26 & 1.29 & 2 & $10^{-5}$ & 0.17 & 1.66 \\
\hline
\end{tabular}

Table II: JET data main inputs. Pulse 85308 time averaged over $10.35 \mathrm{~s}-10.85 \mathrm{~s}$ 


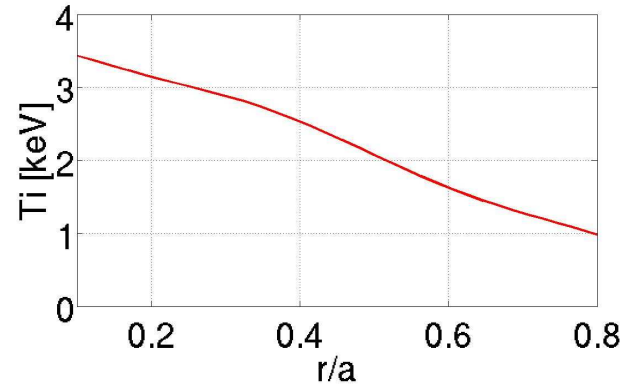

(a) Ion temperature profile

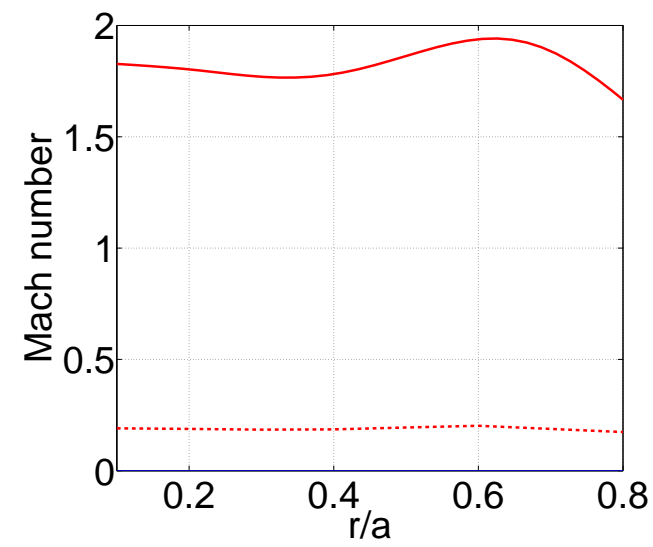

(c) Impurity (here Tungsten W, solid line) and Deu-(d terium (dashed line) Mach number

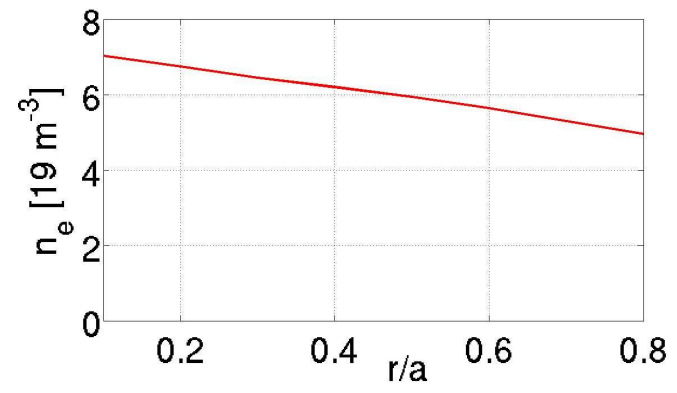

(b) Electron density profile

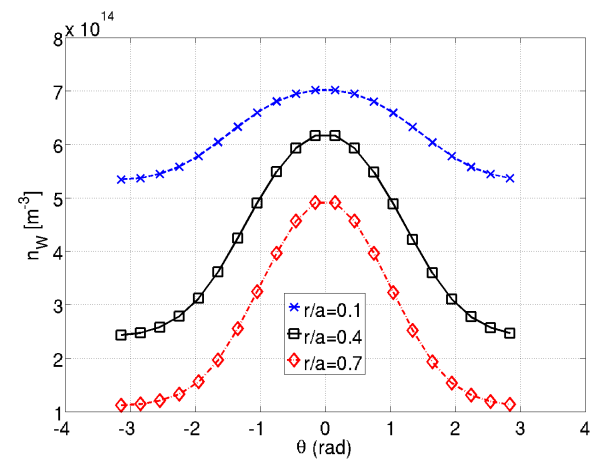

-(d) W distribution vs the poloidal angle $\theta$ from NEO simulations for $\mathrm{r} / \mathrm{a}=0.1$ (blue,cross) 0.4 (dark, square) 0.7 (red, diamond)

Figure 2: Input profiles based on JET 85308 baseline H-mode shot $\left(I_{P}=2.5 M A\right.$ and $\left.\mathrm{B}=2.7 \mathrm{~T}\right)$ for simulated timeslice (averaged over $\mathrm{t}=10.35 \mathrm{~s}-10.85 \mathrm{~s})$. Experimental data and error bars can be found in [6].

The Mach number is defined as $M=\sqrt{\frac{m \omega^{2} \mathrm{R}^{2}}{2 T}}$ where $\mathrm{m}$ is the mass of the considered species, $\mathrm{R}$ is the major radius, $\mathrm{T}$ the temperature and $\omega$ is the angular frequency. Mach number from figure 2c) is computed with NEO based on experimental angular frequency and temperature.

Collisionality parameter is defined as $\nu^{*}=\frac{q R \nu}{v_{t h} \epsilon^{3 / 2}}$. For Deuterium, the dominant collision frequency is $\nu_{D D}=$ $\frac{4 \sqrt{\pi}}{3} \frac{n_{D} e^{4} \ln \Lambda}{\epsilon_{0}^{2} m_{D}^{1 / 2} T_{D}^{3 / 2}}$. For, W, the dominant collision frequency is $\nu_{W D}=\frac{8 \sqrt{\pi}}{3 \sqrt{2}} \frac{n_{D} Z_{W}^{2} e^{4} l n \Lambda}{\epsilon_{0}^{2} m_{W} m_{D}^{-1 / 2} T_{D}^{3 / 2}}$. Both definitions are based on equations (7)-(9) and (B.30) from [23].

Figure 2d) shows that poloidal asymmetries are stronger at the edge. This comes from the dependency on the local value of $\mathrm{R}$ at a given poloidal angle $R_{\text {local }}$ in the expression of the $\mathrm{W}$ density presented in [6] and re-written in equation (21) in the case of isotropic temperature :

$$
n_{Z}(\theta)=n_{Z 0} \exp \left(-\frac{e Z \phi(\theta)}{T_{i}}+\frac{m_{Z} \omega^{2}\left(R_{\text {local }}(\theta)^{2}-R_{0}^{2}\right)}{2 T_{i}}\right)
$$

With $n_{Z 0}$ the impurity density at the low field side, $R_{\text {local }}$ is the local major radius and $R_{0}$ its value at the low field side, $\omega$ is the angular frequency, $\theta$ is the poloidal angle, $\mathrm{Z}$ is the impurity charge, $m_{Z}$ the impurity mass, and $\Phi$ a poloidally varying potential. 


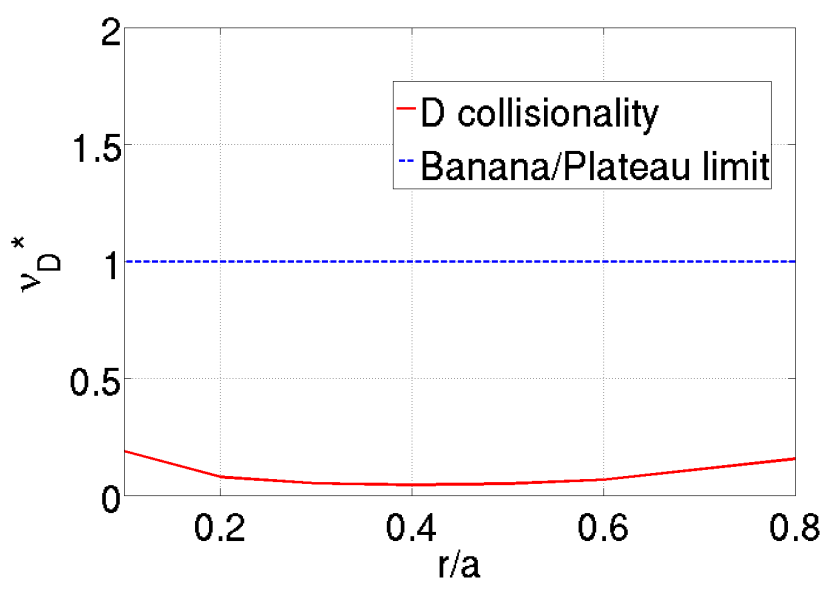

(a) D collisionality

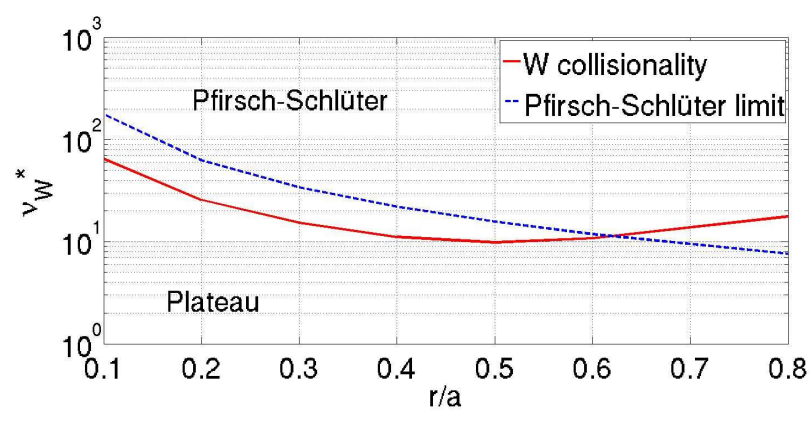

(b) W collisionality

Figure 3: D and W collisionalities calculated from JET-like 85308 profiles from figure 2.

Figure 3a) illustrates the D collisionality and the limit banana-plateau regime. Deuterium remains deep in the banana regime across the whole plasma. On figure $3 \mathrm{~b}$ ), the dashed line corresponds to the limit above which $\mathrm{W}$ reaches the Pfirsch-Schlüter regime. One can see that $\mathrm{W}$ is in Pfirsch-Schlüter regime only at $\mathrm{r} / \mathrm{a}>0.6$. Therefore $\mathrm{r} / \mathrm{a}>0.6$ is the only region where both species are in the regimes (W trace and Pfirsch-Schlüter, D banana) where the Angioni and Helander formula from [18] strictly applies. In the next section we compare $P_{A}^{\text {model }}$ and $Q_{B}^{\text {model }}$ with NEO coefficients and we study the impact of the theoretical assumptions.

B. $\quad P_{A}$ and $Q_{B}$ terms

$P_{A}$ and $Q_{B}$ terms were extracted as explained in the previous section. On figure $4, \mathrm{H}$ corresponds to the WenzelSigmar formulation from equation (4). For the studied JET-ILW case, $\mathrm{H}$ stays very close to $-0,5$. 


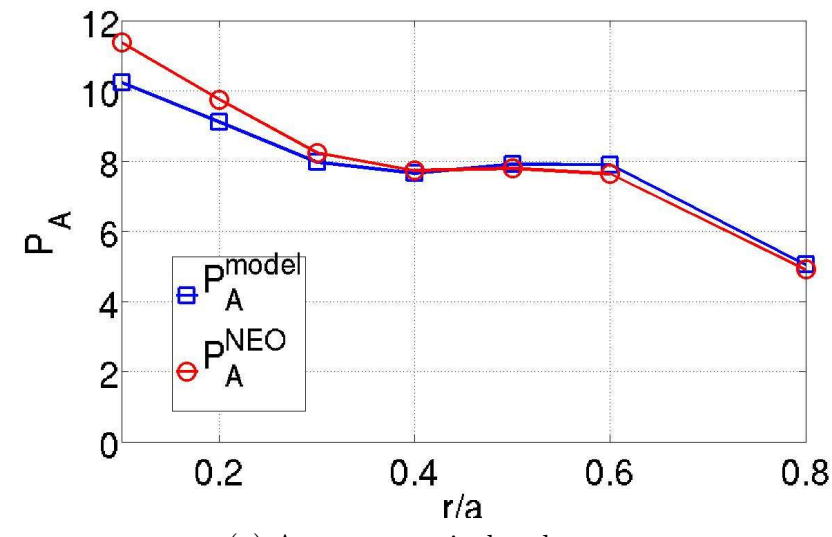

(a) Asymmetry pinch enhancement

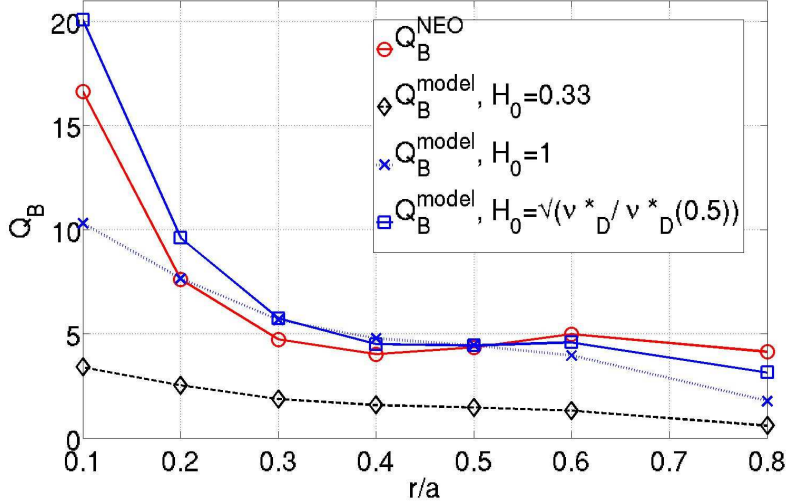

(b) Temperature screening reduction

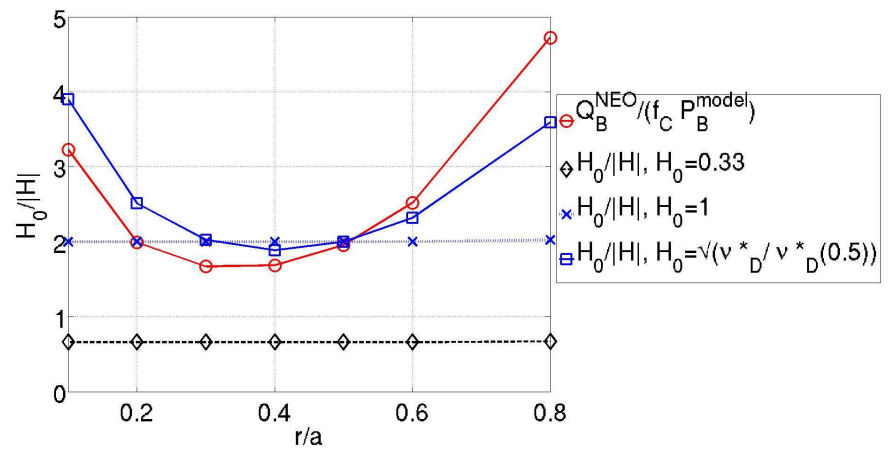

(c) Ratio of temperature screening coefficients

Figure 4: Comparison of NEO results and model results for JET data pulse n ${ }^{\circ} 85308$

On figure 4a) one can see that globally $P_{A}^{\text {model }}$ shows a good agreement with NEO, which is very encouraging because, as seen in figure 3), not all the assumptions of the analytical derivation are well fulfilled in this pulse. However, one can note that at $r / a=0.1-0.3, P_{A}^{\text {model }}$ underestimates $P_{A}^{N E O}$. This is likely due to having $\mathrm{W}$ in plateau where the analytical formula does not strictly apply. Figure $4 \mathrm{~b}$ ) shows $Q_{B}^{N E O}$ compared with different $Q_{B}^{\text {model }}$ combinations, each with a different $H_{0}$ formulation. Figure 4c) shows the numerical value of $H_{0} /|H|$ extracted : $Q_{B}^{N E O}$ is divided by $f_{C} P_{B}^{\text {model }}$, assuming that equation (7) is an accurate description of NEO results. $|H|$ corresponds to Wenzel-Sigmar coefficient from equation (6). On figure $4 \mathrm{~b}$ ) one can see that $Q_{B}^{\text {model }}$ with $H_{0}=0.33$ as initially proposed by Angioni-Helander in [18] (dashed line and diamond) is well below $Q_{B}^{N E O}$ (circles and solid line). $Q_{B}^{\text {model }}$ with $H_{0}=1$ (crosses and dot line) seems to be a better approximation, but at low r/a the fit can be improved. The best fit out of the three $H_{0}$ formulations presented here seems to be the empirical choice of $H_{0}=\sqrt{\nu_{D} * / \nu_{D} *(0.5)}$, with $\nu *_{D}(0.5)$ the value of $\mathrm{D}$ main ion collisionality at $\mathrm{r} / \mathrm{a}=0,5$ (solid line and squares). The collisionality dependence allows to simultaneously reproduce the increase of $H_{0} /|H|$ in the center and towards the last closed flux surface, shown on figure $4 \mathrm{c}$ ). The normalisation of main ion collisionality at $\mathrm{r} / \mathrm{a}=0,5$ was chosen because of the excellent agreement between $Q_{B}^{N E O}$ and $Q_{B}^{\text {model }}$. Indeed, according to [17], the 0.33 value was calculated with a simplified collision operator and remains valid only with $\mathrm{W}$ in Pfirsch-Schlüter regime and $\mathrm{D}$ in banana regime, for large temperature and density gradients. One can see that $H_{0}=1$ is a better fit to the numerical results than 0.33 , but it mismatches at small $\mathrm{r} / \mathrm{a}$ and $\mathrm{r} / \mathrm{a}>0.6$. The $H_{0}$ formulation depending on the collisionality gives a better match. However, a more physics based formulation for $H_{0}$ is required instead of an adjusted formulation depending on the normalization value.

To summarize, there is no radial range where both species are in the regimes (W trace and Pfirsch-Schlüter, D banana) where the Angioni and Helander formula from [18] strictly applies. However analytical $P_{A}^{\text {model }}$ and $Q_{B}^{\text {model }}$ show a very good agreement with NEO coefficients, provided a collisionality dependence is introduced in $H_{0}$. In order to study the validity of the formula out of its limits, and try to quantify its reliability, in Appendix B we study the impact of $\mathrm{W}$ Mach number on the analytical formula. The results show that for $\mathrm{W}$ Mach numbers $>2 P_{A}^{\text {model }}$ overestimates NEO results (up to $50 \%$ ), impacting also $Q_{B}^{\text {model }}$ term. But for this JET-ILW case, as shown on figure 2c) W Mach number is low enough so that analytical coefficients can be used. 


\section{Reconstruction of transport coefficients using NCLASS}

To complete the test of the validity of the model, one can compare NEO coefficients from equations (15)-(16) with a combination of NCLASS runs and geometrical $P_{A}^{\text {model }}$ and $P_{B}^{\text {model }}$ from equations (2) and (3). NCLASS inside JETTO provides a factor 1100 speedup compared with NEO because it is a fluid-moment based model, solving a lower dimensionality problem, while NEO solves exactly the drift kinetic equation. Moreover, NCLASS uses a simple collision operator, which saves time compared to NEO especially with multi-species cases, like the JET-ILW pulse used here.

For clarity, the three transport coefficients are presented as they would be used in a transport code : the diffusion term, defined in equation (12); the main ion gradient pinch velocity term, defined in equation (10); and the temperature screening term, defined in equation (11). But instead of using the analytical expressions from equations (10)-(12), we use NCLASS poloidally symmetric transport coefficients $D_{s y m}^{N C L A S S}, V_{N, s y m}^{N C L A S}$ and $V_{T, s y m}^{N C L A S}$, obtained by using the same procedure shown in equations (15)-(16). Therefore we define, in equations (22)-(24), coefficients that combine NCLASS poloidally symmetric coefficients and analytical $P_{A}$ and $P_{B}: D_{\text {asym }}^{N C L S S, \text { model }}, V_{N, \text { asym }}^{N C L A S S, m o d e l}$ and $V_{T, \text { asym }}^{\text {NCLAS }, \text { model }}$.

$$
\begin{gathered}
D_{\text {asym }}^{\text {NCLAS }, \text { model }}=D_{\text {sym }}^{N C L A S S} P_{A}^{\text {model }} \\
V_{N, \text { asym }}^{\text {NCLASS,model }}=Z D_{\text {sym }}^{N C L A S S} P_{A}^{\text {model }} \frac{R}{L_{n i}} \\
V_{T, \text { asym }}^{\text {NCLASS,model }}=V_{T, \text { sym }}^{N C L A S S}\left[P_{A}^{\text {model }}+\frac{H_{0} f_{C} P_{B}^{\text {model }}}{H}\right]
\end{gathered}
$$

For the comparison between NCLASS and NEO we also introduce transport coefficients which are a combination of NEO poloidally symmetric coefficients associated with $P_{A}^{\text {model }}$ and $P_{B}^{\text {model }}$, defined as follows :

$$
\begin{gathered}
D_{\text {asym }}^{N E O, \text { model }}=D_{\text {sym }}^{N E O} P_{A}^{\text {model }} \\
V_{N, \text { asym }}^{N E O, \text { model }}=Z D_{\text {sym }}^{N E O} P_{A}^{\text {model }} \frac{R}{L_{n i}} \\
V_{T, \text { asym }}^{N E O, \text { model }}=V_{T, \text { sym }}^{N E O}\left[P_{A}^{\text {model }}+\frac{H_{0} f_{C} P_{B}^{\text {model }}}{H}\right]
\end{gathered}
$$




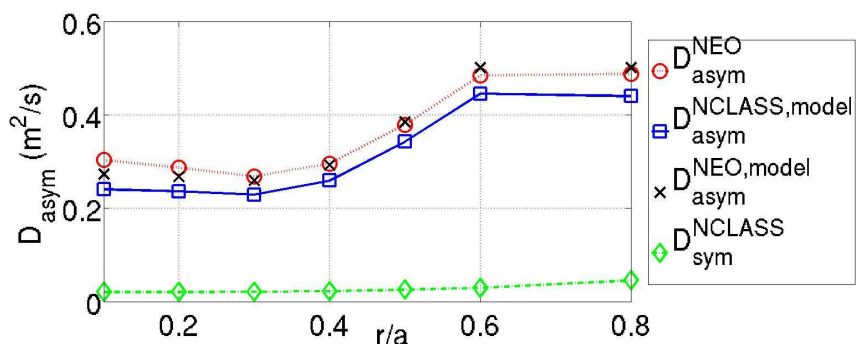

(a) Diffusion term

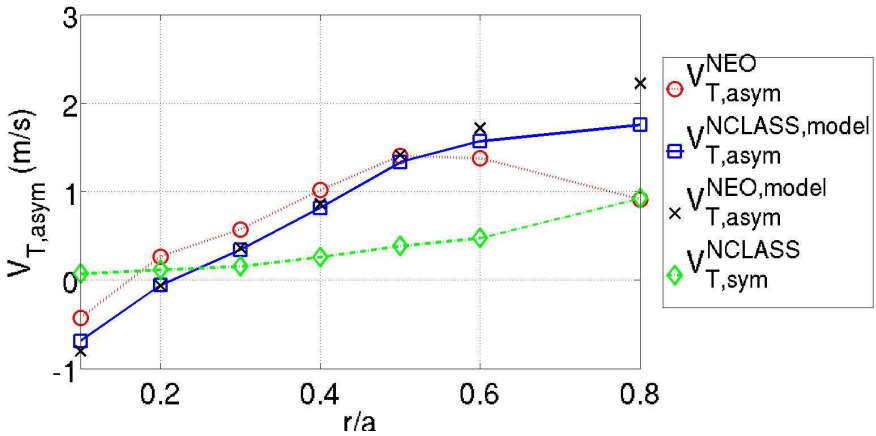

(c) Temperature screening term

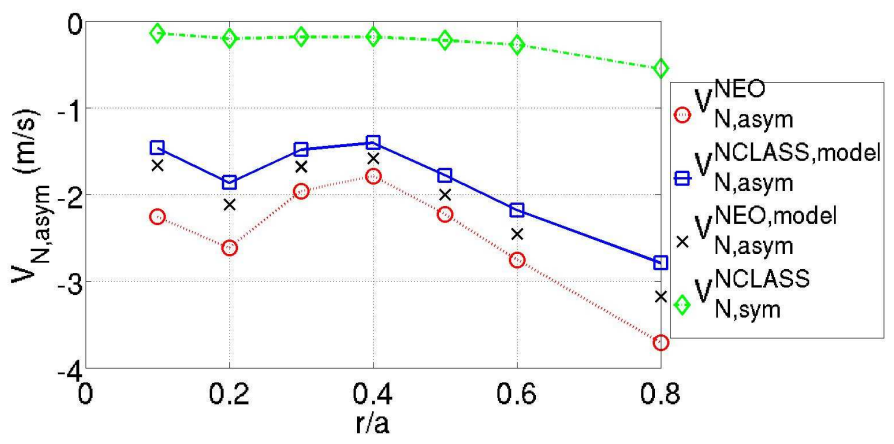

(b) D density driven pinch term

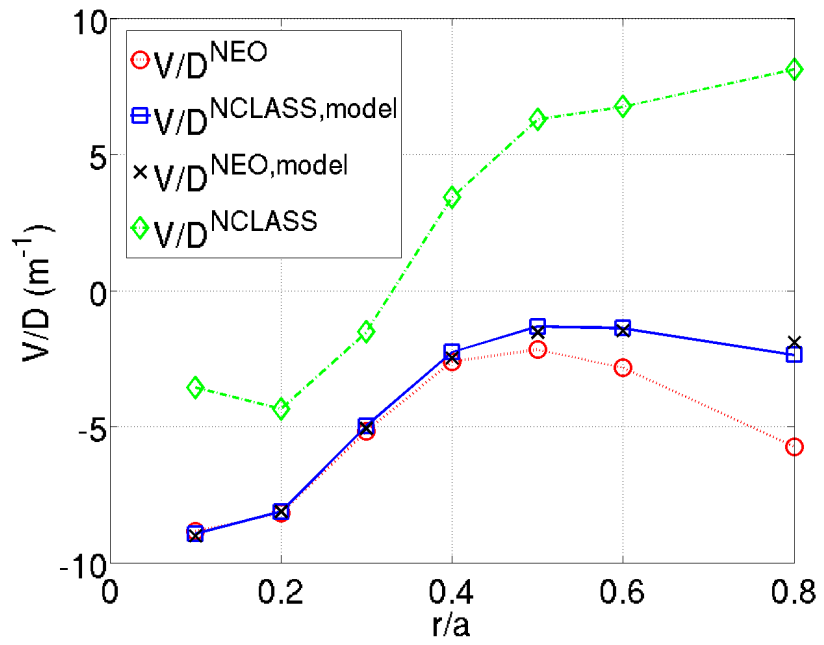

(d) $\mathrm{V} / \mathrm{D}$ ratio $V_{T, \text { asym }}+V_{N, \text { asym }} / D_{\text {asym }}$

Figure 5: Reconstruction of $\mathrm{W}$ transport coefficients : comparison between NEO and NCLASS+correction factors

First of all, one can notice on figures 5 that NCLASS alone (dash-dot line and diamonds) underestimates all transport coefficients by an order of magnitude for $M_{W} \sim 2$. On the contrary NCLASS combined with $P_{A}^{\text {model }}$ and $P_{B}^{\text {model }}$ (squares and solid line) is very close to NEO (circles and dot line) coefficients while obtained 1100 times faster. Indeed NEO runs take the same time, with or without asymmetry. NEO and $P_{A}^{\text {model }}$ and $P_{B}^{\text {model }}$ combined with NEO (crosses) symmetric runs give also globaly similar results.

Regarding the diffusion term (figure 5a), $D_{s y m}^{N C L A S S} \times P_{A}^{\text {model }}$ (squares and full line) is lower than both NEO-only diffusion coefficient (circles and dotted line), and NEO symmetric combined with $P_{A}^{\text {model }}$ (crosses). The difference in collision operator between NEO and NCLASS explains the gap between $D_{\text {sym }}^{N C A S S^{A}} \times P_{A}^{\text {model }}$ and NEO symmetric combined with $P_{A}^{\text {model }}$. Finally the difference between NEO symmetric combined with $P_{A}^{\text {model }}$ and NEO-only diffusion coefficient comes from the difference between $P_{A}^{m o d e l}$ and $P_{A}^{N E O}$ on figure 4 . We mostly want to see how $D_{s y m}^{N C L A S S} \times$ $P_{A}^{\text {model }}$ compares with NEO-only diffusion coefficient and although we recover the difference between $P_{A}^{\text {model }}$ and $P_{A}^{N E O}$ on figure 4 , both coefficients remain comparable.

The fit on the D density driven pinch term on figure 5)b) shows a similar trend : NCLASS combined with analytical $P_{A}^{\text {model }}$ and $Q_{B}^{\text {model }}$ is lower than NEO-only coefficient. One can notice that this term is one order of magnitude bigger than the diffusion term in figure $5 \mathrm{a}$ ), due to the $\mathrm{W}$ charge factor.

Concerning the temperature screening term on figure 5c) we use $H_{0}=\sqrt{\nu_{D} *_{\nu_{* D}(0.5)}}$ as it seems to be the best adjustment for this case. On figure 5c), there is a sign change at low epsilon, coming from $H_{0}$ and $\mathrm{H}$ compensating each other. The agreement between NEO and NCLASS combined with analytical $P_{A}^{\text {model }}$ and $Q_{B}^{\text {model }}$ is very good except for the last point at $\mathrm{r} / \mathrm{a}=0.8$ where a factor 2 discrepancy is observed. However one can notice that the temperature asymmetry screening term is two times weaker than the $\mathrm{D}$ density driven pinch contribution to the $\mathrm{W}$ flux.

Figure $5 \mathrm{~d}$ shows the ratio $\mathrm{V} / \mathrm{D}$, ie the ratio of the convection term (combining $V_{T}$ and $V_{N}$ ) to the diffusion term 
D. One can see that NCLASS alone (green dash-dot lines and diamond) overestimates the peaking by one order of magnitude. The agreement between NEO alone (red circles), NEO combined with $\mathrm{Pa} / \mathrm{Pb}$ model (black crosses) and with NCLASS combined with $\mathrm{Pa} / \mathrm{Pb}$ model (bleu squares) is excellent from the center until $\mathrm{r} / \mathrm{a}=0.4$. For $\mathrm{r} / \mathrm{a}>0.4$ the combination of $\mathrm{Pa} / \mathrm{Pb}$ model with NCLASS or NEO over-estimates the peaking by a maximum of $50 \%$ at $\mathrm{r} / \mathrm{a}=0.8$. This difference comes mainly from the overestimation of the temperature screening term (see figure 5c).

The last step is to test the combination NCLASS + geometric $P_{A}^{\text {model }}$ and $Q_{B}^{\text {model }}$ inside a JET transport solver, JETTO [13]. The goal is to compare the resulting W 2D profile time evolution with $P_{A}^{\text {model }}$ and $Q_{B}^{\text {model }}$ and full NEO with poloidal asymmetries.

\section{APPLICATION IN JETTO}

$P_{A}^{\text {model }}$ and $Q_{B}^{\text {model }}$ equations are implemented in the JET integrated modeling platform JETTO [13]. JETTO is coupled with NCLASS and NEO, but also with the impurity module SANCO [26], that calculates impurity profiles and the amount of radiation produced. We compare on figure 6 the $\mathrm{W}$ profile calculated with NEO inside JETTO, and the W profile calculated using NCLASS inside JETTO associated with geometric $P_{A}^{\text {model }}$ and $Q_{B}^{\text {model }}$. To calculate analytically $P_{A}^{\text {model }}$ and $Q_{B}^{\text {model }}$, the fraction of passing particles is outputted from JETTO magnetic equilibrium. The $\mathrm{W}$ distribution is calculated inside JETTO using equation (21) above. Therefore, all the pieces necessary to estimate $P_{A}^{\text {model }}$ and $Q_{B}^{\text {model }}$ are computed independently from NEO. The simulations starting at $\mathrm{t}=10.35 \mathrm{~s}$ are performed over $0,5 \mathrm{~s}$ so that all codes are converged, with one set of interpretive background profiles. Only neoclassical transport profile is evolved. The input profiles used in JETTO are based on the JET pulse 85308 illustrated on figure 2 . In order to test the neoclassical $\mathrm{W}$ transport, the $\mathrm{W}$ turbulent transport is artificially reduced. NCLASS simulations with $P_{A}^{\text {model }}$ and $Q_{B}^{\text {model }}$ ran on average 1100 times faster compared with NEO simulations, as found when comparing stand alone NEO vs NCLASS combined with $P_{A}^{\text {model }}$ and $Q_{B}^{\text {model }}$ in section III.

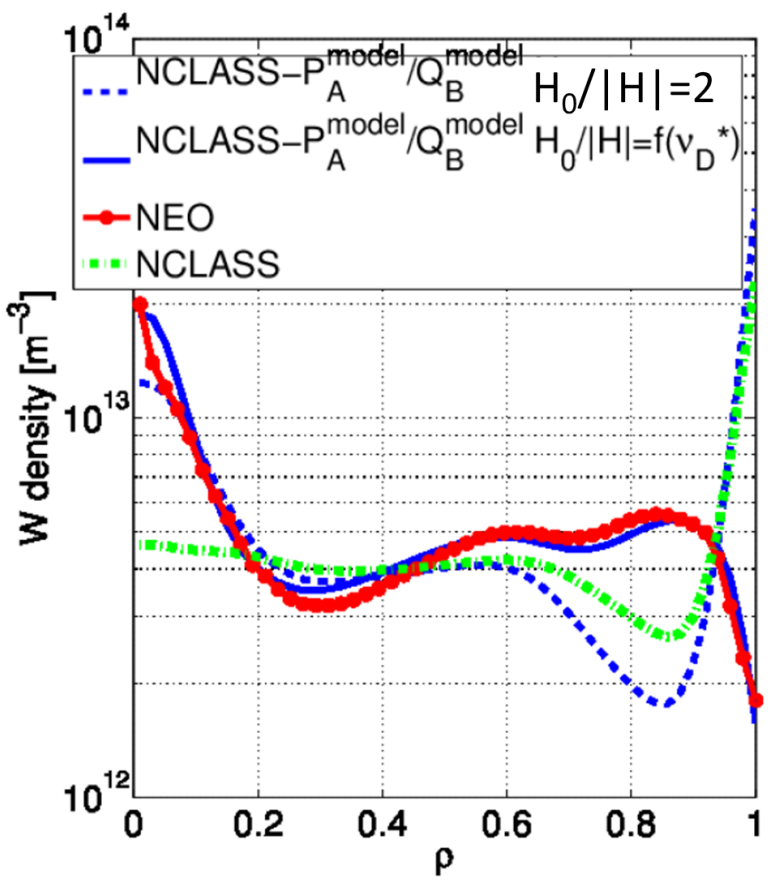

Figure 6: W density profile depending on $\rho$ : comparison between NEO and NCLASS $+P_{A}^{\text {model }} / P_{B}^{\text {model }}$ inside JETTO based on JET case 85308 (time averaged over $\mathrm{t}=10.35 \mathrm{~s}-10.85 \mathrm{~s}$ ).

On figure 6 , the predicted $\mathrm{W}$ density profiles by NEO and by NCLASS $+P_{A}^{\text {model }} / Q_{B}^{\text {model }}$ are compared. One can see that for the whole $\mathrm{r} /$ a range NEO (full line and circles) and NCLASS $+P_{A}^{\text {model }} A / Q_{B}^{\text {mode }} P_{\text {with }} H_{0}$ depending on $\nu_{D} *$ (full line) are very close. The agreement of NEO with and NCLASS $+P_{A}^{\text {model }} / Q_{B}^{\text {model }}$ with $H_{0} /|H|=2$ (dashed line) is also very good within $\rho=0.5$, except in the very core where NCLASS $+P_{A}^{\text {model }} / Q_{B}^{\text {model }}$ with $H_{0} /|H|=2$ underestimates the peaking. NCLASS (dashed and dotted line) does not capture the $\mathrm{W}$ core density peaking. For $\rho$ larger than 0.5 up to the pedestal region, the curves split in two groups : NCLASS $+P_{A}^{\text {model }} / Q_{B}^{\text {model }}$ with $H_{0}$ depending on $\nu_{D} *$ stays in very good agreement with NEO. However in this region NCLASS alone and NCLASS $+P_{A}^{\text {model }} / Q_{B}^{\text {model }}$ 


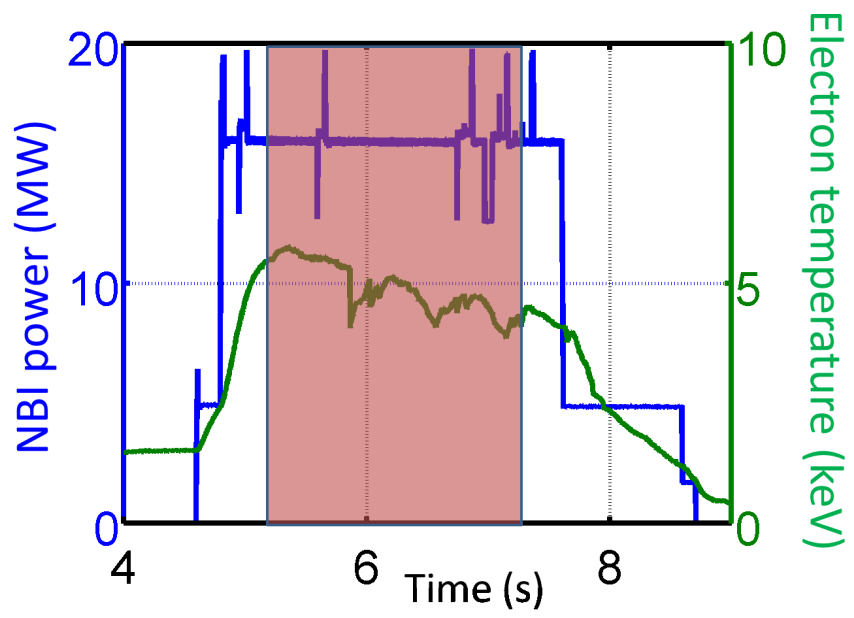

Figure 7: Experimental timetraces of NBI, $T_{e}(\mathrm{ECE})$ of 82722 pulse

with $H_{0} /|H|=2$ both underestimate NEO results. In the pedestal region the study made in section II.b) does not apply. NCLASS alone and NCLASS $+P_{A}^{\text {model }} / Q_{B}^{\text {model }}$ with $H_{0} /|H|=1$ both present an unrealistic peaking at the boundary. NCLASS $+P_{A}^{\text {model }} / Q_{B}^{\text {model }}$ with $H_{0}$ depending on $\nu_{D} *$ keeps its very good agreement with NEO. Therefore a $H_{0}$ increase with $\nu_{D} *$ increasing seems to be a key ingredient to predict the $\mathrm{W}$ profile. The underlying physics still requires to be investigated.

To further test the robustness of $P_{A}^{\text {model }}$ and $Q_{B}^{\text {model }}$ over time, another JET ILW H-mode pulse was chosen. The hybrid pulse $82722\left(B_{T}=2 \mathrm{~T} I_{P}=1.7 \mathrm{MA}\right)$ shows $\mathrm{W}$ accumulation (see figure $8 \mathrm{a}$ ) therefore it is important to see if the formula reproduces successfully this pattern. The simulation runs for $1.6 \mathrm{~s}$, from $5.5 \mathrm{~s}$ to $7.1 \mathrm{~s}$, which corresponds to the shaded part on figure 7. In this time window only Neutral Beam Injection is at play. We also have several sawteeth (seen on the central temperature figure 7) and Edge Localized Modes. The main plasma profiles evolution (electron density and temperature, ion temperature and plasma rotation) is not evolved, instead we use fits based on experimental data. Those profiles are updated every 0.1s. Only neoclassical transport is evolved using NEO or the $P_{A}^{\text {model }} / Q_{B}^{\text {model }}$ formula combined with NCLASS. At the beginning of the simulation $n_{W} / n_{D}=5.10^{-5}$, is chosen to match experimental radiation level, assumed to be caused by $\mathrm{W}$ only. Be is added to match experimental $Z_{\text {eff }}=1.34$. For this JETTO simulation we only used the $H_{0}$ depending on $\nu_{D} *$ formulation. The goal of the simulation is to compare the ability of NEO and $P_{A}^{\text {model }} / Q_{B}^{\text {model }}$ to reproduce the $\mathrm{W}$ experimental behavior.

In order to quantify more precisely the quality of the simulations, the $\mathrm{W}$ density timetraces of the last 0.9 seconds of JETTO simulation are shown at $\mathrm{r} / \mathrm{a}=0.01$ (on figure $8 \mathrm{a}$ ) and $\mathrm{r} / \mathrm{a}=0.5$ (on figure $8 \mathrm{~b}$ ). One can see that at $\mathrm{r} / \mathrm{a}=0.01$ on figure 8a, very close to the plasma center, NCLASS combined with $P_{A}^{\text {model }} / Q_{B}^{\text {model }}$ simulation (blue dot dashed line) overestimates NEO prediction (red full solid line) by a factor 5. JETTO with NEO simulation also overestimates the experimental $\mathrm{W}$ content (dark dashed line) and misses the strong $\mathrm{W}$ density decreases due to sawtooth crashes. However NEO captures the accumulation occuring from $6.8 \mathrm{~s}$ to $7.1 \mathrm{~s}$. At mid-radius on figure $8 \mathrm{~b}$, NEO and NCLASS combined with $P_{A}^{\text {model }} / Q_{B}^{\text {model }}$ simulations reach a much better agreement, with a maximum of $30 \%$ difference. However both simulations overestimate the experimental $\mathrm{W}$ content up to a factor 3 . Figure $8 \mathrm{c}$ ) shows the $\mathrm{W}$ density profile at $\mathrm{t}=7.1 \mathrm{~s}$ after $1.6 \mathrm{~s}$ of simulation. One can see that both NEO and NCLASS combined with $P_{A}^{\text {model }} / Q_{B}^{\text {model }}$ simulations overestimates the $\mathrm{W}$ density from $\mathrm{r} / \mathrm{a}=0.25$ outward. NEO captures the central peaking quite well while NCLASS combined with $P_{A}^{\text {model }} / Q_{B}^{\text {model }}$ overestimates it by a factor 4 . One can notice that the estimated W density remains flat from $\mathrm{r} / \mathrm{a}=0.8$ outwards, this is a boundary condition in the pedestal region.

$2 \mathrm{D} \mathrm{W}$ density maps at $\mathrm{t}=6.8 \mathrm{~s}$, therefore after $1.3 \mathrm{~s}$ of simulation, are shown on figure 9 . Figure $9 \mathrm{a}$ shows the prediction of JETTO with NCLASS and $P_{A}^{\text {model }} / Q_{B}^{\text {model }}$, figure $9 \mathrm{~b}$ ) shows the prediction of JETTO with NEO, and figure 9c) shows the experimental W density estimated from SXR and UV. The poloidal asymmetries are clearly visible on figure 9c), the $\mathrm{W}$ did not move towards the center yet. One can see that JETTO with NEO prediction is very close to the experimental $\mathrm{W}$ distribution the $\mathrm{W}$ density absolute values are comparable $\left(10^{15}\right.$ for the experimental data versus 1.8.0 ${ }^{15}$ for JETTO simulation). Some W moved already in the center. Indeed this can be caused by the fact that the simulation start with a flat $\mathrm{W}$ profile, therefore there is some $\mathrm{W}$ in the center from the start. The JETTO simulation with NCLASS and $P_{A}^{\text {model }} / Q_{B}^{\text {model }}$ predicts that all the $\mathrm{W}$ already moved toward the center, over-predicting the $\mathrm{W}$ content by a factor 5 , even though the poloidal asymmetry is still present (light shape on figure $9 \mathrm{a})$. 


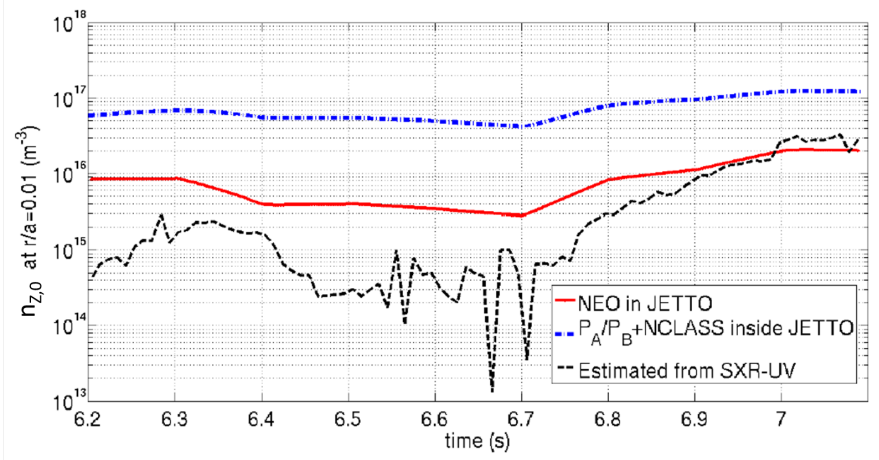

(a) $\mathrm{W}$ density over time at $\mathrm{r} / \mathrm{a}=0.01$

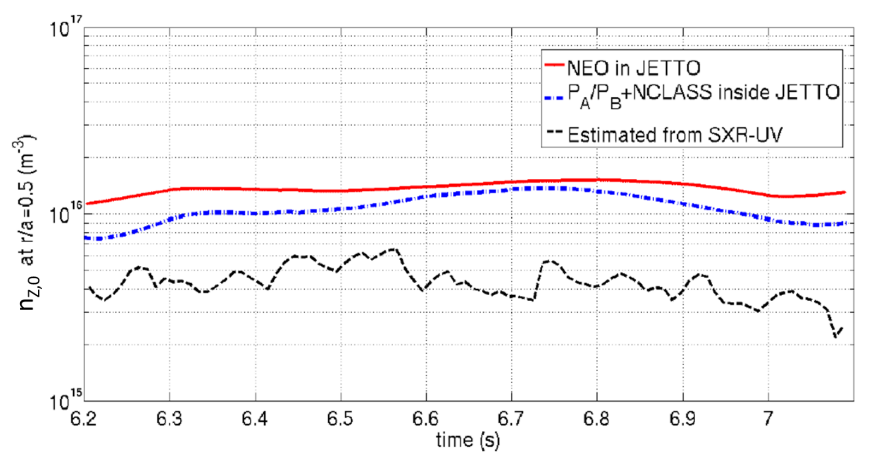

(b) $\mathrm{W}$ density over time at $\mathrm{r} / \mathrm{a}=0.5$

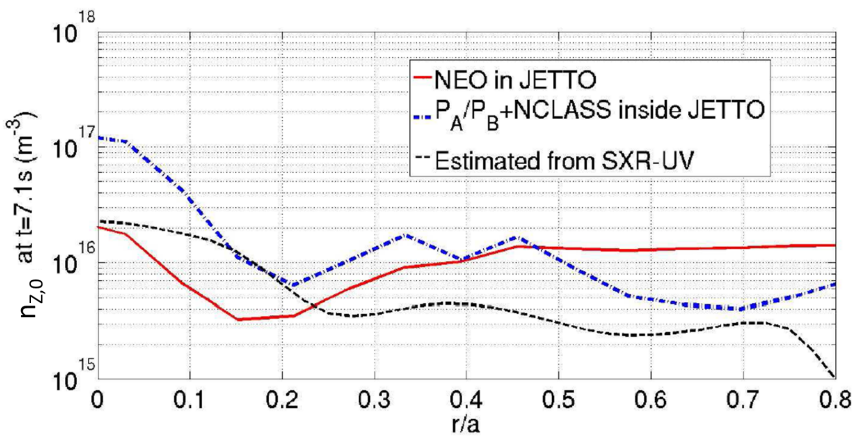

(c) $\mathrm{W}$ density profile at $\mathrm{t}=7.1 \mathrm{~s}$ after $1.6 \mathrm{~s}$ of simulation

Figure 8: $\mathrm{W}$ density at the Low Field Side over time at $\mathrm{r} / \mathrm{a}=0.01$ and $\mathrm{r} / \mathrm{a}=0.5$ and $\mathrm{W}$ density profile at the Low

Field Side at $\mathrm{t}=7.1 \mathrm{~s}$

To summarize, NCLASS associated with the equations $P_{A}^{\text {model }}$ and $Q_{B}^{\text {model }}$ allows to reproduce poorly the W density predicted by NEO in JETTO, while being 1100 times faster. It especially overestimates the core accumulation up to a factor 5 . The choice of the collisionality dependency of $H_{0}$ formulation was proved to be crucial to reproduce of NEO results. On a time evolving simulation, NCLASS and $P_{A}^{\text {model }} / Q_{B}^{\text {model }}$ predictions captures correctly the poloidal asymmetries but overestimates the neoclassical transport in the center. In order to be able to use $P_{A}^{\text {model } /}$ $Q_{B}^{\text {model }}$ for integrated modeling, the $H_{0}$ formulation must be revisited, either thanks to a better empirical regression or thanks to a theory based model.

\section{CONCLUSIONS}

Due to its large mass and charge, Tungsten neoclassical transport can be significantly enhanced by poloidal asymmetries [18]. Poloidal asymmetries are produced by the centrifugal force in presence of NBI $[5,14,16,17,27]$ and/or by $\mathrm{RF}$ heating $[8,28,29]$. In some JET cases, the enhancement can reach an order of magnitude (first established 


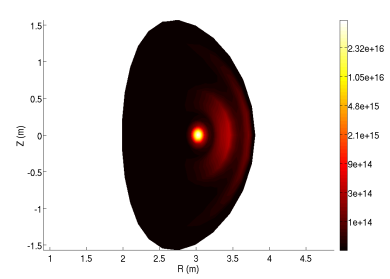

(a) $\mathrm{Pa} / \mathrm{Pb}$ model + NCLASS

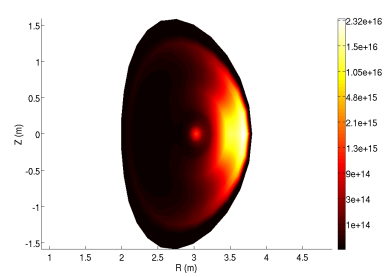

(b) NEO

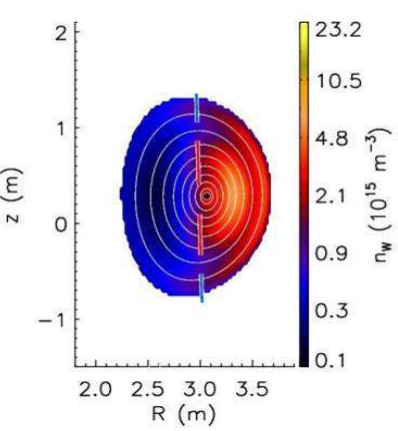

(c) Estimation from SXR-UV measurements

Figure 9: Comparison of $2 \mathrm{D}$ plots of $\mathrm{W}$ density at $\mathrm{t}=6.8 \mathrm{~s}$ (after $1.3 \mathrm{~s}$ of simulation) for 82722

in [16] and continued in $[5,6,22])$. It is therefore essential to take poloidal asymmetries into account in integrated modeling. The effect of poloidal asymmetries are captured by the neoclassical code NEO. However, NEO leads to significant computational expense in integrated modelling applications. The goal of this paper was to determine if it was feasible to combine geometric analytical terms from [18] describing the effect of poloidal asymmetries on $\mathrm{W}$ flux, with a simpler and faster neoclassical code, NCLASS [19], in order to produce results similar to the ones obtained with NEO.

At first, the analytical formula by Angioni-Helander [18] was tested outside of its range of applicability. Indeed, W can be in the plateau regime and not only in Pfirsch-Schlüter regime for realistic tokamak plasma parameters. The analytical formula has been empirically adjusted to match better NEO by accounting for a collisionality dependence in the reduction factor of the temperature screening due to poloidal asymetries. Towards the magnetic axis, W collisionality in plateau leads to slightly under estimated convection enhancement factor, which sightly impacts the reconstruction of the transport coefficients. As long as the D is in the banana regime, the analytic formula can be applied even with $\mathrm{W}$ out of the Pfisch-Schlüter regime. For very strong poloidal asymmetries with main ions in the banana regime, the geometric convection enhancement factor overestimates NEO results (up to 50\%), impacting also temperature screening term.

The neoclassical code, NCLASS has been combined with the analytical geometric formula and implemented in JETTO. In one case based on JET baseline scenario parameters, this fast model can predict the same $\mathrm{W}$ density profile as NEO while saving a factor of a thousand in computer time. In a second JET hybrid scenario case, the central tungsten peaking was overestimated due to a sensitive balance between temperature screening and inward impurity convection near the magnetic axis. The model in its present formulation therefore cannot be considered to have general applicability, but could be used for scoping studies before running the full NEO model. The parametric dependencies of the temperature screening reduction due to poloidal asymmetrics still remains to be better characterised and explained by neoclassical theory; such an understanding would allow our fast neoclassical model for heavy impurity transport to be extended to more general applicability. In order to gain CPU time while keeping the accuracy of NEO, another possibility would be the use of Neural Networks regression as done for QuaLiKiz [30, 31].

For future devices such as ITER and DEMO, neoclassical transport is expected to be much less dominant than in present devices thanks to reducedx angular momentum input. This would have beneficial effects on $\mathrm{W}$ behavior, as presented in [32]. The results obtained in this paper show the potential of a faster model in integrated modelling applications, saving significant computational time in W-transport simulations in the presence of poloidal asymmetries, compared to full NEO calculations.

\section{Appendix A: NEO inputs and outputs}

EQUILIBRIUM_MODEL=3 corresponds to the General Grad-Shrafranov equilibrium. COLLISION_MODEL=4 corresponds to the use of the Full Linearized Fokker-Plancl operator. N_ENERGY=10 corresponds to the number of energy Laguerre polynomials. N_RADIAL $=1$ corresponds to the number of radial positions. N_XI=19 corresponds to the number of Legendre polynomials. ROTATION_MODEL $=2$ means that toroidal rotation effects are included. $\mathrm{N}_{-}$THETA $=21$ correspond to the number of theta gridpoints.

These simulations are run with 5 species, with N_SPECIES $=5$ : Deuterium $\left(n^{\circ} 1\right)$, electrons $\left(n^{\circ} 2\right)$, hydrogen $\left(n^{\circ} 3\right)$, 


\begin{tabular}{|c|c|c|c|c|c|c|c|}
\hline r/a & 0.1 & 0.2 & 0.3 & 0.4 & 0.5 & 0.6 & 0.8 \\
\hline \hline Z_4 & 44 & 43 & 42 & 38 & 34 & 31 & 26 \\
\hline DLNNDR & 0,30 & 0,46 & 0,43 & 0,44 & 0,53 & 0,62 & 0,90 \\
\hline DLNTDR & 0,75 & 0,86 & 1,02 & 1,66 & 2,30 & 2,53 & 3,27 \\
\hline RMAJ_OVER_A & 3,11 & 3,11 & 3,11 & 3,11 & 3,10 & 3,09 & 3,07 \\
\hline Q & 0,90 & 0.93 & 0,98 & 1,10 & 1,17 & 1,33 & 2,02 \\
\hline SHEAR & $2,1.10^{-2}$ & $8,2.10^{-2}$ & 0,19 & 0,35 & 0,58 & 0,92 & 2,17 \\
\hline OMEGA_ROT & $8,1.10^{-2}$ & $7,8.10^{-2}$ & $7,5.10^{-2}$ & $7,3.10^{-2}$ & $7,4.10^{-2}$ & $7,6.10^{-2}$ & $6,2.10^{-2}$ \\
\hline OMEGA_ROT_DERIV & $-6,4.10^{-2}$ & $-7,2.10^{-2}$ & $-6,7.10^{-2}$ & $-6,1.10^{-2}$ & $-6,8.10^{-2}$ & $-0,10$ & $-0,22$ \\
\hline RHO_STAR & $4,1.10^{-3}$ & $3,9.10^{-3}$ & $3,7.10^{-3}$ & $3,4.10^{-3}$ & $3,0.10^{-3}$ & $2,6.10^{-3}$ & $1,7.10^{-3}$ \\
\hline NU_1 & $9,2.10^{-4}$ & $1,0.10^{-3}$ & $1,2.10^{-3}$ & $1,5.10^{-3}$ & $2,1.10^{-3}$ & $3,1.10^{-3}$ & $7,2.10^{-3}$ \\
\hline
\end{tabular}

Table III: Full list of NEO inputs

\begin{tabular}{|c|c|c|c|c|c|c|c|}
\hline $\mathrm{r} / \mathrm{a}$ & 0.1 & 0.2 & 0.3 & 0.4 & 0.5 & 0.6 & 0.8 \\
\hline \hline$<n_{W}>\left(m^{-3}\right)$ & $6,15.10^{14}$ & $5,31.10^{14}$ & $4,67.10^{14}$ & $4,09.10^{14}$ & $3,46.10^{14}$ & $2,91.10^{14}$ & $2,57.10^{14}$ \\
\hline$f_{c}$ & 0,74 & 0,64 & 0,56 & 0,50 & 0,45 & 0,41 & 0,33 \\
\hline$\Gamma_{W}\left(m^{-2} s^{-1}\right)$ & $-1,47.10^{15}$ & $-1,19.10^{15}$ & $-5,69.10^{14}$ & $-1,96.10^{14}$ & $-1,39.10^{14}$ & $-2,45.10^{14}$ & $-6,03.10^{14}$ \\
\hline
\end{tabular}

Table IV: Selection of NEO outputs

W ( $\left.n^{\circ} 4\right)$, Berylium $\left(n^{\circ} 5\right)$. Masses and temperatures are nomalized to the Deuterium mass. The Deuterium charge, mass and density stay unchanged at all radii : MASS_1 $1=1, Z_{-} 1=1$ and DENS_1 $=0.79$. The electrons charge, mass and density stay unchanged at all radii : MASS $\_2=2,72 \cdot 10^{-3}, \mathrm{Z}_{-} 2=-1$, DENS $\_2=1$. The hydrogen charge, mass and density stay unchanged at all radii : MASS_3 $=0.5, \mathrm{Z} \_3=1$, DENS_3 $=9,5.10^{-2}$. The $\mathrm{W}$ mass and density stay unchanged at all radii : MASS_4 $=92$, DENS_4 $=1.10^{-5}$. The Berylium charge, mass and density stay unchanged at all radii : MASS_5 $=4.5$, Z_5 $=4$, DENS_5 $=2,9.10^{-2}$. All species have the same temperature (TEMP=1), the same density gradient DLNNDR $=-a^{\delta l n n} / \delta r$ and DLNTDR $=-a^{\delta l n T} / \delta r . \quad$ NU_1 $=\frac{\nu_{i i}}{v_{\text {norm } / a}}$ with $\nu_{i i}=\frac{\sqrt{2} \pi e^{4} Z_{i}^{4} n_{i}}{m_{i}^{1 / 2} T_{i}^{3 / 2}} \ln \Lambda$ and $v_{\text {norm }}=\sqrt{T i / m_{i}}$. SHEAR is defined as $s=\frac{r}{q} \frac{\delta q}{\delta r}$. RMAJ_OVER_A is the ratio of the flux-surface-center major radius to the normalizing length scale a. Q is the safety factor. OMEGA_ROT is the angular toroidal frequency defined as $\frac{\omega_{0}}{v_{\text {norm }} / a}$. OMEGA_ROT_DERIV is the normalized toroidal rotation shear defined as $\frac{d \omega_{0}}{d r} \frac{a^{2}}{v_{\text {norm }}}$. RHO_STAR is the normalized Larmor radius : $\rho^{*}=\frac{c \sqrt{m_{D} T_{D}}}{e B} \frac{1}{a}$.

These specific NEO inputs were used in the scan shown on figures 3 in Section III.

In the board below are listed main NEO outputs relevant for this work :

\section{Appendix B: Mach number dependence}

Another assumption needs to be studied : the Mach number dependency. Note that Mach number for main ions, $M_{i}$, for the case $T_{i}=T_{W}$, is proportional to $M_{W}$ by definition, $M_{i}=M_{W} \sqrt{m_{i} / m_{W}}$. In the $M_{W}$ scan we consider values up to $M_{W} \sim 3$, observed in JET NBI pulses [5]. In this range the bulk ion Mach number remains small, $M_{i}<0.3$. Figures 10 illustrate such a scan where both $\mathrm{W}$ and the main ion (here Deuterium) Mach numbers are simultaneously varied.

One can see that the agreement is very good until $M_{Z} \approx 2$; above, NEO does not increase as much as geometric terms predict, both for $P_{A}$ and $Q_{B}$ terms with both $H_{0}$ formulations, showing a discrepancy up to $50 \%$. Note that for $\mathrm{W}$ Mach number down to zero, the values of the neoclassical theory in absence of poloidal asymmetries are recovered, namely $P_{A}=1$ and $Q_{B}=0$. The dependence in Mach number is quadratic, the impact of poloidal asymmetries becomes significant from $M_{Z} \approx 0.5$. In figure 11 , another scan of the $\mathrm{W}$ Mach number was made, but this time $\mathrm{W}$ is placed in the Pfirsch-Schlüter regime. To do so, we increased both $\mathrm{D}$ and $\mathrm{W}$ densities, keeping the ratio $n_{W} / n_{D}=10^{-5}$ fixed so that $\mathrm{W}$ is still a trace impurity. In the configuration shown on figure 11, $\mathrm{W}$ is in Pfirsch-Schlüter and $\mathrm{D}$ is still in banana regime.

On figure 11 the fits are almost perfect for $P_{A}$ until $M_{Z} \approx 2$, with a maximum error of $7 \%$ compared with $20 \%$ on figure 10a. This can be explained by the fact that, with $\mathrm{W}$ in Pfisch-Schlüter, NEO values are increased. On figure 


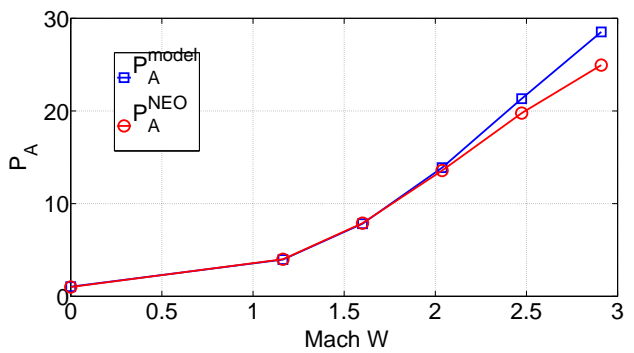

(a) Asymmetry pinch enhancement

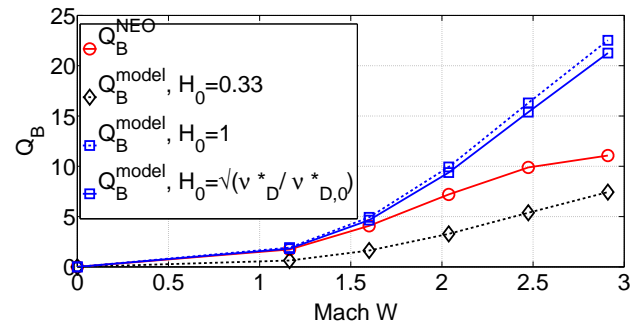

(b) Temperature screening reduction

Figure 10: $P_{A}$ and $Q_{B}$ terms depending on W Mach number. JET data shot $\mathrm{n}^{\circ} 85308, \mathrm{r} / \mathrm{a}=0.4$ in table 1 except $n_{W} / n_{D}=10^{-6}$. D in banana and $\mathrm{W}$ in plateau. For the last two points, NEO resolution increased to 41 theta gridpoints and 39 extensions in Legendre polynomials.

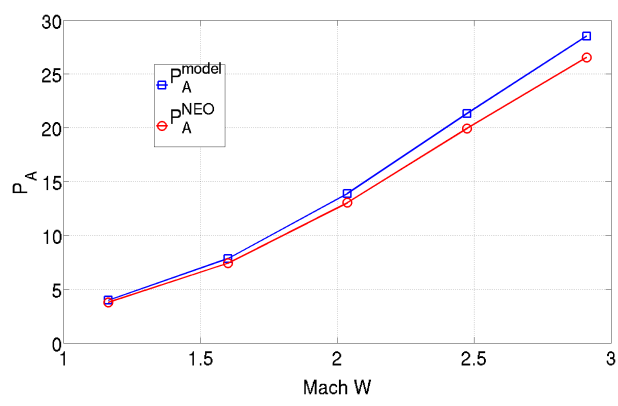

(a) Asymmetry pinch enhancement

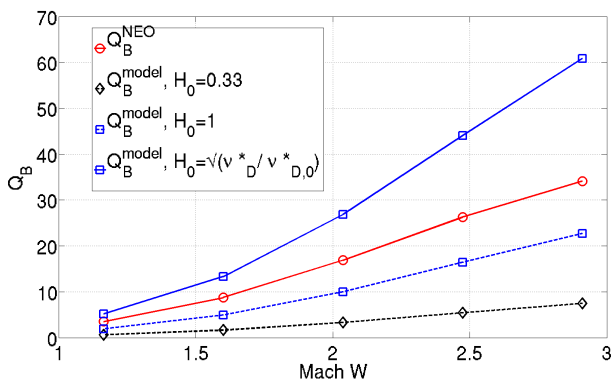

(b) Temperature screening reduction

Figure 11: $P_{A}$ and $Q_{B}$ terms depending on W Mach number. JET data shot $n^{\circ} 85308, n_{W} / n_{D}=10^{-6} \mathrm{r} / \mathrm{a}=0.4$ in table 1 except $n_{D}=5.10^{20}$. D in banana and $\mathrm{W}$ forced in Pfirsch-Schlüter regime

$11 \mathrm{~b}$ for the $Q_{B}$ part, one can see that forcing W into Pfirsch-Schlüter increased $Q_{B}^{N E O}$ up to a factor 3. This brings $Q_{B}^{N E O}$ closer to $Q_{B}^{\text {model }}$ with $H_{0}=1$ formulation. Indeed $Q_{B}^{\text {model }}$ with $H_{0}=1$ values were not impacted by the change in $\mathrm{W}$ collisionality from figure $10 \mathrm{~b}$ to figure $11 \mathrm{~b}$. However, increasing the $\mathrm{D}$ content as well as the $\mathrm{W}$ content in order to keep the ratio $n_{W} / n_{D}=10^{-5}$ did change the value of the $H_{0}$ formulation depending on $\nu_{D}^{*}$, which shifted the $Q_{B}^{\text {model }}$ accordingly. This reinforces the importance to find be better characterised $H_{0}$ formulation.

For very strong poloidal asymmetries, W needs to be in Pfirsch-Schlüter regime for the formula to be applicable. In this configuration the $H_{0}$ formulation plays a crucial role in the estimation of $Q_{B}^{\text {model }}$. Therefore, until a better $H_{0}$ formulation is established, the use of NEO is recommended.

\section{ACKNOWLEDGMENTS}

"This work has been carried out within the framework of the EUROfusion Consortium and has received funding from the Euratom research and training programme 2014-2018 under grant agreement No 633053. The views and opinions expressed herein do not necessarily reflect those of the European Commission."

With the financial support of la Région Provence-Alpes-Côte d'Azur

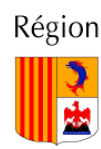

Provence-Alpes-Côte d'Azur 
[1] S. P. Hirshman and D.J. Sigmar. Neoclassical transport of impurities in tokamak plasmas. Nucl. Fusion, $1079,1981$.

[2] P. Helander and D.J. Sigmar. Collisional Transport in Magnetized Plasmas. 2002.

[3] C. Angioni, L. Carraro, T. Dannert, N. Dubuit, R. Dux, C. Fuchs, X. Garbet, L. Garzotti, C. Giroud, R. Guirlet, F. Jenko, O. J W F Kardaun, L. Lauro-Taroni, P. Mantica, M. Maslov, V. Naulin, R. Neu, A. G. Peeters, G. Pereverzev, M. E. Puiatti, T. Pütterich, J. Stober, M. Valovi, M. Valisa, H. Weisen, and A. Zabolotsky. Particle and impurity transport in the Axial Symmetric Divertor Experiment Upgrade and the Joint European Torus, experimental observations and theoretical understanding. Physics of Plasmas, 14(5):1-9, 2007.

[4] T. Fülöp, S. Braun, and I. Pusztai. Impurity transport driven by ion temperature gradient turbulence in tokamak plasmas. Physics of Plasmas, 17(6):1-9, 2010.

[5] C Angioni, P Mantica, T Pütterich, M Valisa, M Baruzzo, E A Belli, P Belo, F J Casson, C Challis, P Drewelow, C Giroud, N Hawkes, T C Hender, J Hobirk, T Koskela, L Lauro Taroni, C F Maggi, J Mlynar, T Odstrcil, M L Reinke, M Romanelli, and J E T EFDA Contributors. Tungsten transport in JET H-mode plasmas in hybrid scenario, experimental observations and modelling. Nuclear Fusion, 54(8):83028, 2014.

[6] F. J. Casson, C. Angioni, E. A. Belli, R. Bilato, P. Mantica, T. Odstrcil, T. Puetterich, M. Valisa, L. Garzotti, C. Giroud, J. Hobirk, C. F. Maggi, J. Mlynar, M. L. Reinke, JET EFDA Contributors, and ASDEX-Upgrade Team. Theoretical description of heavy impurity transport and its application to the modelling of tungsten in JET and ASDEX Upgrade. pages 1-9, 2014.

[7] M L Reinke. Poloidal variation of high- Z impurity density due to hydrogen minority ion cyclotron resonance heating on Alcator.

[8] R. Bilato, O. Maj, and C. Angioni. Modelling the influence of temperature anisotropies on poloidal asymmetries of density in the core of rotating plasmas. Nuclear Fusion, 54(7):072003, 2014.

[9] E A Belli and J Candy. Kinetic calculation of neoclassical transport including self-consistent electron and impurity dynamics. Plasma Phys. Control. Fusion, 50(9):95010, 2008.

[10] E A Belli and J Candy. Full linearized Fokker Planck collisions in neoclassical transport simulations. Plasma Phys. Control. Fusion, 54(54):15015-17, 2012.

[11] C. Bourdelle, X. Garbet, F. Imbeaux, A. Casati, N. Dubuit, R. Guirlet, and T. Parisot. A new gyrokinetic quasilinear transport model applied to particle transport in tokamak plasmas. Physics of Plasmas, 14(11):1-13, 2007.

[12] G. M. Staebler, J. E. Kinsey, and R. E. Waltz. A theory-based transport model with comprehensive physics. Physics of Plasmas, 14(5):1-7, 2007.

[13] Michele Romanelli, Gerard Corrigan, Vassili Parail, Sven Wiesen, Roberto Ambrosino, Paula Da, Silva Aresta, Luca Garzotti, Derek Harting, Florian Köchl, Tuomas Koskela, Laura Lauro-taroni, Chiara Marchetto, Massimiliano Mattei, Elina Militello-asp, Maria Filomena, Ferreira Nave, Stanislas Pamela, Antti Salmi, Pär Strand, Association Euratom-fzj, Forschungszentrum Jülich, and D Jülich. JINTRAC : A System of Codes for Integrated Simulation of. 9:1-4, 2014.

[14] J. A. Wesson. Poloidal distribution of impurities in a rotating tokamak plasma. Nuclear Fusion, 37(5):577, 1997.

[15] Per Helander. Neoclassical transport in a rotating impure plasma. Physics of Plasmas, 5(4):1209-1211, 1998.

[16] M. Romanelli and M. Ottaviani. Effects of density asymmetries on heavy impurity transport in a rotating tokamak plasma. Plasma Phys. Control. Fusion, 1767, 1998.

[17] T. Fulop and P. Helander. Nonlinear neoclassical transport in a rotating impure plasma with large gradients. Doktorsavhandlingar vid Chalmers Tekniska Hogskola, 3066(1538):3066-3075, 1999.

[18] C Angioni and P Helander. Neoclassical transport of heavy impurities with poloidally asymmetric density distribution in tokamaks. Plasma Physics and Controlled Fusion, 56(12):124001-124008, 2014.

[19] W. a. Houlberg, K. C. Shaing, S. P. Hirshman, and M. C. Zarnstorff. Bootstrap current and neoclassical transport in tokamaks of arbitrary collisionality and aspect ratio. Physics of Plasmas, 4(9):3230, 1997.

[20] T. Fülöp and P. Helander. Nonlinear neoclassical transport in toroidal edge plasmas. Contributions to Plasma Physics, 42(2-4):339-349, 2002.

[21] E A Belli, J Candy, and C Angioni. Pfirsch-Schluter neoclassical heavy impurity transport in a rotating plasma. Plasma Physics and Controlled Fusion, 56(12):124002, 2014.

[22] C. Angioni, F. J. Casson, P. Mantica, T. Pütterich, M. Valisa, E. A. Belli, R. Bilato, C. Giroud, and P. Helander. The impact of poloidal asymmetries on tungsten transport in the core of JET H-mode plasmas. Physics of Plasmas, 22(5), 2015.

[23] K.W. Wenzel and D.J. Sigmar. Neoclassical analysis of impurity transport following transition to improved particle confinement. Nuclear Fusion, 30(6):1117-1127, 1990.

[24] E A Belli and J Candy. An Eulerian method for the solution of the multi-species drift-kinetic equation. Plasma Physics and Controlled Fusion, 51(7):75018, 2009.

[25] P. H. Rutherford. Impurity transport in the Pfirsch-Schluter regime. Physics of Fluids, 17(9):1782, 1974.

[26] B Alper, R Giannella, K Lawson, F Marcus, M Mattioli, P Smeulders, and M Von Hellermann. Impurity Transport of High Performance Discharges in JET. pages 3-7.

[27] F. L. Hinton and S. K. Wong. Neoclassical ion transport in rotating axisymmetric plasmas. Physics of Fluids, 28(10):3082, 1985.

[28] W Choe, C S Chang, and M Ono. Temperature anisotropy in a cyclotron resonance heated tokamak plasma and the generation of poloidal electric field. Physics of Plasmas, 2:2044-2054, 1995. 
[29] Ye O Kazakov, I Pusztai, T Fülöp, and T Johnson. Poloidal asymmetries due to ion cyclotron resonance heating. Plasma Physics and Controlled Fusion, 54(10):105010, 2012.

[30] J Citrin, F Felici A Teplukhina, C Bourdelle, S Breton, F Imbeaux, J Redondo, and O Sauter. First multi-channel core transport simulations with RAPTOR using a neural network transport model. 2017.

[31] K L Van De Plassche, J Citrin, C Bourdelle, V Dagnelie, and A Ho. Realtime capable quasilinear gyrokinetic modelling using neural networks. EPS, 2017.

[32] C Angioni, M Sertoli, R Bilato, V Bobkov, A Loarte, R Ochoukov, T Odstrcil, T Pütterich, J Stober, The Asdex, and Upgrade Team. A comparison of the impact of central ECRH and central ICRH on the tungsten behaviour in ASDEX Upgrade H-mode plasmas. Nucl. Fusion, 57(056015), 2017. 